\title{
Hamilton-Jacobi theory for constrained systems
}

\author{
D. Dominici and G. Longhi \\ Dipartimento di Fisica, Università di Firenze, Istituto Nazionale di Fisica Nucleare, Sezione di Firenze, \\ Firenze, Italy \\ J. Gomis and J. M. Pons \\ Departament de Física Teòrica, Universitat de Barcelona, Barcelona, Spain
}

(Received 13 October 1982; accepted for publication 6 January 1984)

\begin{abstract}
We extend the Hamilton-Jacobi formulation to constrained dynamical systems. The discussion covers both the case of first-class constraints alone and that of first- and second-class constraints combined. The Hamilton-Dirac equations are recovered as characteristic of the system of partial differential equations satisfied by the Hamilton-Jacobi function.
\end{abstract}

PACS numbers: 03.20. + i, 03.30. + p, 12.90. + b

\section{INTRODUCTION}

In recent years the old problem of a consistent formulation of relativistic Hamiltonian dynamics, for a system of particles in direct interaction, has received new interest. ${ }^{1,2}$ It is now widely recognized that, in order to establish manifest covariance, it is convenient to look for a formulation in terms of constrained dynamics, where the constraints on the phase-space variables guarantee the correct number of physical degrees of freedom, and essentially contain the dynamics of the system under consideration.

From this point of view, relativistic Hamiltonian dynamics can be seen as a theory of systems constrained in phase space. Such a theory was developed by Dirac ${ }^{3}$ and reformulated in various respects by others, ${ }^{4}$ so by now it seems to be a well-developed subject.

The corresponding Hamilton-Jacobi theory has been the subject of various papers, mainly with regard to theoretical field applications. ${ }^{5}$ What seems to be lacking in the literature on this subject, in the opinion of the present authors, is a unified systematic approach to the Hamilton-Jacobi theory for a system of particles, especially with regard to the possible applications in the presence of second-class constraints.

For this reason, the present work reviews the Hamilton-Jacobi method in a systematic and almost didactic way, presenting in some detail even those topics which are well known from the mathematical literature on systems of partial differential equations.

In order to give a unified approach for both first- and second-class constraints, the concept of (Cauchy) characteristic vectors will be used, so that in both cases the equations of motion will appear as characteristic equations. In this way Dirac's bracket structure will emerge naturally, and the integrability conditions of the characteristic system will be explicitly verified by using the properly generalized Jacobi identity.

With regard to this last point, the Mayer identity (that is, the generalization of the Jacobi identity to nonhomogeneous Poisson brackets) appears to hold for Dirac's brackets also, as has been verified by explicit calculation.

The analysis of the characteristic equations of the given set of constraints, though interesting by itself, is only preliminary to a Hamilton-Jacobi approach. The latter will be given, in the first-class case, by a review of Jacobi's method of integration.

In the second-class case the integrability conditions for the existence of the Hamilton-Jacobi function are not satisfied, so this function, as a function on all phase-space space, does not exist. Nevertheless, as we shall show in Sec. V, in a number of interesting cases, to which, in principle, the problem may always be reduced, the Hamilton-Jacobi method can be used fruitfully to get the solution of the equations of motion.

Since the authors were especially interested in this case, Sec. $V$ is to be considered the central section of this paper. In that section two possible methods are discussed.

The concept of weak equality, first introduced by Dirac, ${ }^{3}$ is used throughout this paper. A careful discussion of its extension to vector fields and differential forms is presented in Appendices A and B.

No applications are discussed in the present work, but the method is best suited for the formulation given by Todorov $^{6}$ and $\mathrm{Komar}^{7}$ of the dynamics of a system of particles, and an application can be found in Ref. 8, where the Hamilton-Jacobi function is calculated for a two-body system. An interesting topic which has not been discussed here, but which deserves further investigation, is the study of symmetries from this point of view.

The paper is organized as follows: In Sec. II we make a general discussion of the extension of the Hamilton-Jacobi theory to constrained systems. Section III is devoted to the study of the first-class constraints systems and Secs. IV and $V$ to the case where second-class constraints are also present. In Sec. VI we show how we can recover the Hamilton-Dirac equations. ${ }^{3}$ In Appendix A the problem of classification of constraints into first- and second-class constraints is studied. Finally in Appendices B and C we prove some useful geometric results for our discussion. In Appendix D the transformation properties of the Hamilton-Jacobi function under canonical transformations are briefly reviewed.

\section{A GENERAL DISCUSSION OF THE HAMILTON- JACOBI THEORY FOR CONSTRAINED SYSTEMS}

Let us assume that a dynamical system is described in terms of a canonical Hamiltonian $H_{c}\left(x^{i}, p_{i}\right)$, where the set 
$\left(x^{i}, p_{i}\right), i=1, \ldots, n$, denotes the phase-space variables, with the following Poisson brackets:

$$
\left\{x^{i}, p_{j}\right\}=\delta_{j}^{i}
$$

and a set of constraints $\phi_{\rho}\left(x^{i}, p_{i}\right)=0, \rho=1, \ldots, m \leqslant n$, which in general will be of both first and second class, in the sense used by Dirac. ${ }^{3}$

If a Lagrangian exists, the canonical Hamiltonian $H_{c}$ is known and the functions $\phi_{\rho}$ will be a consequence of the analysis of the Lagrangian equations of motion, ${ }^{4}$ but more generally we may assume that the dynamical system is given in terms of the set of functions $H_{c}$ and $\phi_{\rho}{ }^{9}$

The Hamilton-Jacobi equations for the given system are expected to be in the form

$$
\begin{aligned}
& \frac{\partial S}{\partial x^{0}}+H_{c}\left(x^{i}, p_{j}\right)=0 \\
& (q=1, \ldots, m), \\
& \phi_{\rho}\left(x^{i}, p_{j}\right)=0
\end{aligned}
$$

where

$$
p_{i}=\frac{\partial S}{\partial x^{i}}
$$

and $S=S\left(x^{i}\right)$ is the Hamilton-Jacobi function.

In the next part of this section we will analyze the set of Eqs. (2.2) from the point of view of the general theory of partial differential equations (PDE) (of first order).

Let us write Eqs. (2.2) in a more compact notation. To this end, it is more convenient to work in an enlarged phase space $\left(x^{\alpha}, p_{\beta}\right), \alpha, \beta=0,1, \ldots, n$, where

$$
p_{0}=\frac{\partial S}{\partial x^{0}}
$$

and

$$
\left\{x^{0}, p_{0}\right\}=1,
$$

and we will write the set of Eqs. (2.2) as

$$
\begin{aligned}
& \phi_{\rho}\left(x^{\alpha}, p_{\alpha}\right)=0 \\
& (\alpha=0,1, \ldots, n, \quad \rho=0,1, \ldots, m),
\end{aligned}
$$

where

$$
\phi_{0}\left(x^{\alpha}, p_{\alpha}\right)=p_{0}+H_{c}\left(x^{i}, p_{i}\right),
$$

and

$$
p_{\alpha}=\frac{\partial S}{\partial x^{\alpha}} .
$$

So the set of equations we want to study is the set (2.6). The choice (2.7) for $\phi_{0}$ will be reserved for the cases where the canonical Hamiltonian $H_{c}$ is different from zero. It is necessary to put some restriction on the functions (2.6), in order to develop the subsequent theory. We make the following assumption about the rank ${ }^{10}$ :

$$
\operatorname{rank}\left\|\frac{\partial \phi_{\rho}}{\partial x^{\alpha}}, \frac{\partial \phi_{\rho}}{\partial p_{\alpha}}\right\|=m+1 .
$$

It is well known from the theory of $\operatorname{PDE}^{12}$ that if $S(x)$ is a solution of Eqs. (2.6), it must also be a solution of the equations

$$
\left\{\phi_{\rho}(x, p), \phi_{\sigma}(x, p)\right\}=0 \quad(\rho, \sigma=0,1, \ldots, m) .
$$

When $\phi_{\rho}$ are a set of first-class constraints, Eqs. (2.10) are satisfied by virtue of Eq. (2.6), but when $\phi_{\rho}$ include second-class constraints, this is no longer true. In this last case we have to add the lhs of Eq. (2.10) as new equations, and continue the procedure until we get a complete system of $\mathrm{PDE}^{12}$ or a set of equations which are inconsistent. (A typical case of this last situation is when the Poisson bracket of two $\phi_{\rho}$ is a constant.)

If we get a complete system, the Hamilton-Jacobi function for this new set will describe a dynamical system different from the original one which was required to satisfy Eq. (2.6) only. The new system will have more constraints, and will describe a completely different physical situation.

As a consequence, when second-class constraints are present, we cannot consider the set (2.6) as a set of HamiltonJacobi equations. Nevertheless, it is known that the characteristic system exists, is completely integrable, and gives the usual Hamilton-Dirac equations of motion ${ }^{3}$ for the system.

In order to construct the characteristic system, we can substitute Eqs. (2.6) with the following exterior differential system $A$ :

$$
A=\left\{\begin{array}{l}
\phi_{\rho} \equiv \phi_{\rho}(x, p)=0 \\
\theta \equiv d S-p_{\alpha} d x^{\alpha}=0
\end{array}\right.
$$

defined in the space $\mathbb{R}^{2(n+1)+1}$ of $(2(n+1)+1)$-tuples

$$
\left(\xi^{a} ; a=1, \ldots, 2 n+3\right) \equiv\left(x^{0}, x^{1}, \ldots, x^{n}, S, p_{0}, p_{1}, \ldots, p_{n}\right) .
$$

According to the usual procedure, ${ }^{13}$ we consider the closure of $A, \bar{A}$ :

$$
\bar{A}=\left\{\begin{array}{c}
\phi_{\rho}=0, \quad d \phi_{\rho}=\left(\frac{\partial \phi_{\rho}}{\partial x^{\alpha}}+p_{\alpha} \frac{\partial \phi_{\rho}}{\partial S}\right) d x^{\alpha} \\
+\frac{\partial \phi_{\rho}}{\partial p_{\alpha}} d p_{\alpha}=0 \\
\theta=0, \quad d \theta=-d p_{\alpha} \wedge d x^{\alpha}=0 .
\end{array}\right.
$$

The characteristic system $C$ of $A$ is the associated Pfaff system of the set $\left\{\theta=0, d \theta=0, d \phi_{\rho}=0\right\}$ and the equations $\phi_{\rho}=0$.

As is shown in Appendix C, for the study of this characteristic system it is convenient to consider the space $Q$ of the vector fields satisfying

$$
i_{v} I_{A} \subseteq I_{A} \text {, }
$$

where $I_{A}$ is the ideal generated by the differential forms $\left\{\theta, d \theta, d \phi_{\rho}\right\},{ }^{13}$ and where the notation $\subsetneq$ means weak inclusion as defined in Appendix $\mathrm{C}$.

By considering Eqs. (2.12) for each of the forms $d \theta, \theta$, and $d \phi_{\rho}$, we get the following results:

$$
\begin{aligned}
& i_{v} d \theta \approx \lambda \rho d \phi_{\rho}+\mu \theta, \\
& i_{v} \theta \approx 0, \\
& i_{v} d \phi_{\rho} \approx 0,
\end{aligned}
$$

where the notation $\approx$ means weak equality as defined in Appendix B.

Using the representation of the vector field in the local coordinates $\xi^{a}$,

$$
v \equiv v^{a} \frac{\partial}{\partial \xi^{a}}=v^{\alpha} \frac{\partial}{\partial x^{\alpha}}+v^{n+1} \frac{\partial}{\partial S}+u_{\alpha} \frac{\partial}{\partial p_{\alpha}}
$$


in Eq. (2.14a), we get

$$
v^{\alpha} d p_{\alpha}-u_{\alpha} d x^{\alpha} \approx \sum_{p=0}^{m} \lambda^{\rho}(\xi) d \phi_{\rho}(\xi)
$$

and

$$
\mu \approx 0 \text {, }
$$

due to the fact that the lhs of Eq. (2.14a) does not contain $d S$.

Taking into account the explicit expression of $d \phi_{\rho}[\mathrm{Eq}$. (2.12)], we have ${ }^{14}$

$$
v^{\alpha}=\lambda \rho \frac{\partial \phi_{\rho}}{\partial p_{\alpha}}, \quad u_{\alpha}=-\lambda^{\rho} \frac{\partial \phi_{\rho}}{\partial x^{\alpha}} .
$$

Equations (2.14b) and (2.14c) give the conditions

$$
\begin{aligned}
& v^{n+1}=p_{\alpha} v^{\alpha}, \\
& v^{\alpha} \frac{\partial \phi_{\rho}}{\partial x^{\alpha}}+u_{\alpha} \frac{\partial \phi_{\rho}}{\partial p_{\alpha}} \approx 0,
\end{aligned}
$$

which, together with Eq. (2.17), determine the components of the characteristic vector fields. Using Eq. (2.17) in Eq. (2.19), we get

$$
\sum_{\sigma=0}^{m} \lambda^{\sigma}\left\{\phi_{\rho}, \phi_{\sigma}\right\} \approx 0
$$

which is a necessary and sufficient condition for the existence of characteristic vector fields.

Let us then discuss Eq. (2.20). We have to consider three different cases corresponding to the value of the rank

$$
r=\operatorname{rank}\left\|\left\{\phi_{\rho}, \phi_{\sigma}\right\}\right\| \quad(\rho, \sigma=0, \ldots, m)
$$

calculated on the manifold defined by Eqs. (2.6):

$$
\begin{aligned}
& \text { (I) } r=0: \lambda^{0}, \ldots, \lambda^{m} \text { arbitrary; } \\
& \text { (II) } 0<r<m+1:(m+1-r) \lambda^{\rho} \text { arbitrary } \\
& (\rho=0,1, \ldots, m-r) ; \\
& \text { (III) } r=m+1: \lambda^{0}=\cdots=\lambda^{m}=0(m+1 \text { even). }
\end{aligned}
$$

Let us recall that $r$ must be even, being the rank of an antisymmetric matrix.

According to the Dirac terminology, case (I) corresponds to a set of first-class constraints, case (II) to a set of first- and second-class constraints, and case (III) to secondclass constraints.

With regard to case (II), the problem arises as to the classification of $\phi_{p}$ in first- and second-class constraints. This problem is solved in Appendix A.

In order to construct the characteristic system, it is necessary to consider the space $Q$ * of 1 -forms weakly incident to $\widetilde{Q}$, i.e.,

$$
i_{v} \bar{\theta} \approx 0, \quad \bar{\theta} \in Q^{*}, v \in \widetilde{Q} .
$$

It is obvious that $\operatorname{dim} Q^{*}=2 n+3-(m+1-r)$.

As suggested in Ref. 13, if the characteristic vectors are defined by a system of equations of the form

$$
v^{a} b_{a}^{b}=0(a, b=1, \ldots, 2 n+3)
$$

for some $b_{a}^{\cdot b}$, then the 1-forms

$$
\bar{\theta}^{a}=d x^{b} b_{b}^{\cdot a}
$$

satisfy Eqs. (2.22). Let us observe that the forms (2.24) will not all be independent, as we will verify in the following.
Nevertheless, using Eqs. (2.24) we will get, with a proper choice, a basis for $Q^{*}$.

\section{THE CHARACTERISTIC SYSTEM AND THE HAMILTON-JACOBI FUNCTION FOR A SET OF FIRST- CLASS CONSTRAINTS}

In this case, corresponding to $r=0$, all the $\lambda$ 's being arbitrary, a basis for the space of the characteristic vectors $\widetilde{Q}$ is given by

$$
\begin{gathered}
v_{\rho}=\frac{\partial \phi_{\rho}}{\partial p_{\alpha}}\left(\frac{\partial}{\partial x^{\alpha}}+p_{\alpha} \frac{\partial}{\partial S}\right)-\frac{\partial \phi_{\rho}}{\partial x^{\alpha}} \frac{\partial}{\partial p_{\alpha}} \\
(\rho=0, \ldots, m),
\end{gathered}
$$

which are linearly independent due to the hypothesis (2.9) on the rank of the matrix of the components of $v_{\rho}$.

By using the nonhomogeneous Poisson bracket ${ }^{15}$

$$
\begin{aligned}
\{f, g\}_{n h}= & \left(\frac{\partial f}{\partial x^{\alpha}}+p_{\alpha} \frac{\partial f}{\partial S}\right) \frac{\partial g}{\partial p_{\alpha}} \\
& -\frac{\partial f}{\partial p_{\alpha}}\left(\frac{\partial g}{\partial x^{\alpha}}+p_{\alpha} \frac{\partial g}{\partial S}\right),
\end{aligned}
$$

we can write the characteristic vectors [Eq. (3.1)] as

$$
v_{\rho}=-\left\{\phi_{\rho},\right\}_{n h} \text {. }
$$

It is now possible to reduce the search for a solution of the system of nonlinear PDE (2.6) to a system of linear homogeneous PDE. In fact, if we consider the system

$$
v_{\rho}(g)=-\left\{\phi_{\rho}, g\right\}_{n h}=0,
$$

where $g: R^{2 n+3} \rightarrow R$, this is a system of linear homogeneous equations which is completely integrable. In fact, using the Mayer identity (Ref. 16, p. 172):

$$
\begin{aligned}
& \left\{f_{1},\left\{f_{2}, f_{3}\right\}_{n h}\right\}_{n h}+\text { cyclic } \\
& =-\frac{\partial f_{1}}{\partial S}\left\{f_{2} f_{3}\right\}_{n h}+\text { cyclic }
\end{aligned}
$$

we have

$$
\begin{aligned}
{\left[v_{\rho}, v_{\sigma}\right](g) \approx } & -\left\{g,\left\{\phi_{\rho}, \phi_{\sigma}\right\}_{n h}\right\}_{n h}-\frac{\partial \phi_{\rho}}{\partial S}\left\{\phi_{\sigma}, g\right\}_{n h} \\
& -\frac{\partial \phi_{\sigma}}{\partial S}\left\{g, \phi_{\rho}\right\}_{n h}-\frac{\partial g}{\partial S}\left\{\phi_{\rho}, \phi_{\sigma}\right\}_{n h},
\end{aligned}
$$

and using the fact that $\phi_{\rho}$ are independent of $S$ and that they are a set of first-class constraints:

$$
\left\{\phi_{\rho}, \phi_{\sigma}\right\}=c_{\rho \sigma \tau} \phi_{\tau},
$$

we finally get

$$
\left[v_{\rho}, v_{\sigma}\right] \approx c_{\rho \sigma \tau} v_{\tau} \quad(\rho, \sigma, \tau=0, \ldots, m),
$$

and, using the results of Appendix C, the system (3.4) is completely integrable over the surface of the constraints.

Thus the system $(3.4)$ has $(2 n+3)-(m+1)$ independent solutions depending on $x, p$, and $S ; m+1$ of these solutions are nothing but the $\phi_{\rho}$ 's, which are functions only of $x$ and $p$.

Among the solutions of the system (3.4), we can choose one, which we will call $G_{1}(x, p, S)$, independent of $\phi_{\rho}$. Then we can add to the set $\phi_{\rho}=0$ the new equations $G_{1}=C_{1}$, where $C_{1}$ is an arbitrary constant, and consider the new set 


$$
v_{\rho}(g)=0, \quad v_{G}(g)=0 .
$$

We can proceed in this way until we have extended the original set of $m+1$ equations to the new set

$$
v_{\rho}(g)=0, \quad v_{G_{1}}(g)=0, \quad \ldots, \quad v_{G_{n-m}}(g)=0,
$$

which is completely integrable and has

$2 n+3-(m+1+n-m)=n+2$ independent solutions, of which $n+1$ are already known: $\phi_{0}, \ldots, \phi_{m}, G_{1}, \ldots, G_{n-m}$. So the system (3.10) still has one new solution, $G_{n-m+1}$. In this way we get an involutory system $\phi_{0}, \ldots, \phi_{m}$,

$G_{1}-C_{1}, \ldots, G_{n-m+1}-C_{n-m+1}$ of $n+2$ functions

$\left(\phi_{\rho}=\phi_{\rho}\left(x^{\alpha}, p_{\alpha}\right), G_{\bar{k}}=G_{\bar{k}}\left(x^{\alpha}, P_{\alpha}, S\right), \rho=0, \ldots, m\right.$,

$\tilde{k}=1, \ldots, n-m+1)$, where $C_{\tilde{k}}$ are $n-m+1$ arbitrary constants.

At this point we can go no further, since if we consider the new set of equations

$$
\left\{\phi_{\rho}, g\right\}_{n h}=0, \quad\left\{G_{\dot{k}}, g\right\}_{n h}=0,
$$

we should conclude that it has just $n+1$ independent solutions $[2 n+3-(m+1+n-m+1)]$, whereas we already know $n+2$ solutions in involution among themselves! This conclusion is wrong, since one of Eqs. (3.11) is not independent, as an equation, from the others, as we will verify at the end of the discussion.

Jacobi's method of integration now involves considering in place of the original set of constraint equations the new set

$$
\begin{aligned}
& \phi_{\rho}=G_{\rho}(x, p)=0 \quad(\rho=0, \ldots, m), \\
& G_{\tilde{k}}(x, p, S)-C_{\tilde{k}}=0 \quad(\tilde{k}=1, \ldots, n-m+1),
\end{aligned}
$$

from which a solution can be obtained algebraically.

In order to show this, we will follow the usual procedure, ${ }^{17}$ here adapted to the use of nonhomogeneous Poisson brackets.

Taking into account Eq. (2.9) and the procedure we have followed, we have the functions $G_{i}(i=0,1, \ldots, n+1)$ independent by construction, so we may assume

$$
\left|\frac{\partial\left(G_{i}\right)}{\partial\left(p_{a}, S\right)}\right| \neq 0
$$

apart from a possible reordering of the canonical variables $(x, p)$; in any case it is essential for at least one of the $G_{i}$ to have the derivative with respect to $S$ different from zero.

It follows that we may solve the equations

$$
G_{i}(x, p, S)-C_{i}=0
$$

(where $C_{\rho}=0, \rho=0,1, \ldots, m$ ) in $p_{a}$ and $S$ :

$$
P_{i} \equiv p_{i}-f_{i}(x, c)=0,
$$

where $P_{n+1}=S$.

The functions $P_{i}$ are in involution with respect to the nonhomogeneous Poisson brackets,

$$
\left\{P_{i}, P_{j}\right\}_{n h} \approx 0 \text {. }
$$

In fact, using (3.12) and (3.15), we have identically with respect to $x^{\alpha}$

$$
G_{i}\left(x^{\alpha}, p_{\alpha}=f_{\alpha}(x, c), S=f_{n+1}(x, c)\right)-C_{i}=0,
$$

and, differentiating in $x^{\alpha}$,

$$
\frac{\partial G_{i}}{\partial x^{\alpha}}+\frac{\partial G_{i}}{\partial p_{\beta}} \frac{\partial f_{\beta}}{\partial x^{\alpha}}+\frac{\partial G_{i}}{\partial S} \frac{\partial f_{n+1}}{\partial x^{\alpha}} \approx 0
$$

or

$$
\frac{\partial G_{i}}{\partial x^{\alpha}} \approx \frac{\partial G_{i}}{\partial p_{\beta}} \frac{\partial P_{\beta}}{\partial x^{\alpha}}+\frac{\partial G_{i}}{\partial S} \frac{\partial P_{n+1}}{\partial x^{\alpha}},
$$

where the weak equality means that the derivatives of $G_{i}$ with respect to $p_{\beta}$ and $S$ must be evaluated on the surface (3.15).

Besides (3.19) we have identically

$$
\frac{\partial G_{i}}{\partial p_{j}}=\frac{\partial G_{i}}{\partial p_{k}} \frac{\partial P_{k}}{\partial p_{j}},
$$

where $j, k=0,1, \ldots, n+1, P_{n+1}=S$.

With (3.19) and (3.20) we have

$$
\left\{G_{i}, G_{j}\right\}_{n h} \approx \frac{\partial G_{i}}{\partial p_{k}} \frac{\partial G_{j}}{\partial p_{h}}\left\{p_{k}-f_{k}(x, c), p_{h}-f_{h}(x, c)\right\}_{n h},
$$

as can easily be verified. Using (3.13), we conclude that

$$
\begin{aligned}
& \left\{P_{k}, P_{h}\right\}_{n h} \approx 0, \\
& \left\{p_{\alpha}-f_{\alpha}(x, c), p_{\beta}-f_{\beta}(x, c)\right\}_{n h} \approx 0, \\
& \left\{p_{\beta}-f_{\beta}(x, c), S-f_{n+1}(x, c)\right\}_{n h} \approx 0,
\end{aligned}
$$

from which we get

$$
\frac{\partial f_{\alpha}(x, c)}{\partial x^{\beta}} \approx \frac{\partial f_{\beta}(x, c)}{\partial x^{\alpha}}
$$

since in $(3.22)$ the bracket reduces to an ordinary Poisson bracket, and

$$
\frac{\partial}{\partial x^{\alpha}} f_{n+1}(x, c)-p_{\alpha} \approx 0 .
$$

This last result shows that $f_{n+1}(x, c)$ is determined by $f_{\alpha}(x, c)$, since the weak equality holds when $p_{\alpha}=f_{\alpha}(x, c)$ and $S=f_{n+1}(x, c)$, that is, we can take

$$
\frac{\partial f_{n+1}(x, c)}{\partial x^{\alpha}}=f_{\alpha}(x, c)
$$

or

$$
d S=f_{\alpha}(x, c) d x^{\alpha}
$$

On the other hand, in (3.24) $p_{\alpha}$ does not appear, so it holds identically and not only when $p_{\alpha}=f_{\alpha}(x, c)$; thus

$$
\frac{\partial f_{\alpha}}{\partial x^{\beta}}=\frac{\partial f_{\beta}}{\partial x^{\alpha}}
$$

which is consistent with $d S$ being an exact form.

For practical purposes it is easier to work with homogeneous (usual) Poisson brackets. In this case we would have to integrate Eq. (3.26) in order to get $S$; this is the way in which this point is usually presented.

Now we may understand the observation made after (3.11). The set of functions $\phi_{\rho}, G_{k}(k=1,2, \ldots, n-m+1)$ are in involution, but the set of equations (3.11) are not independent since, in the new form,

$$
\left\{P_{i}, g\right\}_{n h} \approx 0
$$

[where $\approx$ still means an equality on the surface $(3.15)$ ]; when $i=n+1$, it reads 


$$
\left(p_{\alpha}-\frac{\partial f_{n+1}(x, c)}{\partial x^{\alpha}}\right) \frac{\partial g}{\partial p_{\alpha}} \approx 0
$$

or

$$
\left(f_{\alpha}(x, c)-\frac{\partial f_{n+1}(x, c)}{\partial x^{\alpha}}\right) \frac{\partial g}{\partial p_{\alpha}} \approx 0,
$$

which shows that the last of Eqs. (3.28) is already satisfied. So the real number of independent equations is again $n+1$, with $2 n+3-(n+1)=n+2$ solutions given by $\phi_{\rho}, G_{k}$, or $P_{i}, i=0,1, \ldots, n+1$.

Summing up, in the hypothesis that the set of constraints $\phi_{\rho}$ is first class, a function

$$
S=S(x, c)
$$

exists, which is the solution of Eqs. (2.6). This function is defined apart from an additive constant. Neglecting this constant, $S$ will in general contain $(n+1)-(m+1)$ constants of integration, and so it is a complete integral. It is known from the theory of systems of PDE that from a complete integral it is possible to get all other integrals by means of differentiations and eliminations only. So the Jacobi method of integration gives a general kind of solution.

The constraints (2.6) are contained implicitly in the set of equations (3.15). Indeed, by eliminating $(n+1)-(m+1)$ constants $C_{k}$, we again obtain the constraints (2.6). Finally, let us observe that the transformation

$$
\begin{aligned}
& Q^{\alpha}=x^{\alpha}, \\
& P_{\alpha}=p_{\alpha}-f_{\alpha}(x, c)
\end{aligned}
$$

is a canonical transformation due to Eqs. (3.27). It is a phase transformation generated by the function $S(x, c)$ :

$$
\begin{aligned}
& Q^{\alpha}=e^{-s_{*}} x^{\alpha}=x^{\alpha}, \\
& P_{\alpha}=e^{-s_{*}} p_{\alpha}=p_{\alpha}-f_{\alpha}(x, \mathcal{c}),
\end{aligned}
$$

where the operation $*$ is defined by

$$
e^{A} * B=B+\{A, B\}+\frac{1}{2}(A,\{A, B\}\}+\cdots \text {. }
$$

Let us now continue the discussion on the construction of the characteristic system. As anticipated in Sec. II, the 1forms belonging to the space $Q *$ can be written using Eqs. (2.24), if Eqs. (2.17), (2.18), and (2.19) can be rewritten in the form (2.23). In the hypothesis

$$
\left|\frac{\partial \phi_{\rho}}{\partial p_{\sigma}}\right| \neq 0
$$

by eliminating the functions $\lambda^{\rho}$ in Eqs. (2.17), (2.18), and (2.19), we get

$$
\begin{aligned}
& \lambda^{\rho}=v^{\sigma}\left(A^{-1}\right)_{\sigma}^{\rho}, \\
& A_{\rho}^{\cdot \sigma}=\frac{\partial \phi_{\rho}}{\partial p_{\sigma}}
\end{aligned}
$$

and

$$
\begin{aligned}
& v^{\alpha}-v^{\sigma}\left(A^{-1}\right)_{\sigma}^{\rho} \frac{\partial \phi_{\rho}}{\partial p_{\alpha}}=0 \\
& u_{\alpha}+v^{\sigma}\left(A^{-1}\right)_{\sigma}^{\rho} \frac{\partial \phi_{\rho}}{\partial x^{\alpha}}=0
\end{aligned}
$$

$$
\begin{aligned}
& v^{n+1}-p_{\alpha} v^{\alpha}=0, \\
& v^{\alpha} \frac{\partial \phi_{\rho}}{\partial x^{\alpha}}+u_{\alpha} \frac{\partial \phi_{\rho}}{\partial p_{\alpha}} \approx 0,
\end{aligned}
$$

which are in the form (2.23). By the formal substitution $v^{\alpha} \rightarrow d x^{\alpha}, u_{\alpha} \rightarrow d p_{\alpha}, v^{n+1} \rightarrow d S$, we get the set of 1-forms:

$$
\left\{\bar{\theta}^{\alpha}\right\} \equiv\left\{\begin{array}{l}
\eta^{\alpha}=d x^{\alpha}-d x^{\sigma}\left(A^{-1}\right)_{\sigma}^{\rho} \frac{\partial \phi_{\rho}}{\partial p_{\alpha}} \\
\zeta_{\alpha}=d p_{\alpha}+d x^{\sigma}\left(A^{-1}\right)_{\sigma}^{\cdot \rho} \frac{\partial \phi_{\rho}}{\partial x^{\alpha}} \\
\theta=d S-p_{\alpha} d x^{\alpha} \\
d \phi_{\rho}=d x^{\alpha} \frac{\partial \phi_{\rho}}{\partial x^{\alpha}}+d p_{\alpha} \frac{\partial \phi_{\rho}}{\partial p_{\alpha}}
\end{array}\right.
$$

It is easily verified that only $(2 n+3)-(m+1)$ of these forms are independent and hence can be chosen as a basis for $Q^{*}$. The associated Pfaff system will be given by the exterior differential equations

$$
\begin{aligned}
& d x^{\alpha}=d x^{\sigma}\left(A^{-1}\right)_{\sigma}^{\rho}\left\{x^{\alpha}, \phi_{\rho}\right\}, \\
& d p_{\alpha}=d x^{\sigma}\left(\left.A^{-1}\right|_{\sigma} ^{\rho}\left\{p_{\alpha}, \phi_{\rho}\right\},\right. \\
& d S=p_{\alpha} d x^{\sigma}\left(A^{-1}\right)_{\sigma}^{\rho}\left\{x^{\alpha}, \phi_{\rho}\right\}
\end{aligned}
$$

[where for $\alpha=\sigma$ the first set of equations are identities; see Eqs. (3.34)], to which it is necessary to add the equations

$$
\phi_{\rho}(x, p)=0 \text {, }
$$

in order to get the characteristic system $C$ of our original exterior differential system $A$ [Eqs. (2.11)].

Therefore, since the number of integration constants in (3.30) is $(2 n+3)-(m+1)$ and they must satisfy (3.38), we conclude that the characteristic manifold is parametrized by $2(n-m)+1$ constants.

Observe that the equations $d \phi_{\rho}=0$ are satisfied owing to (3.37) and (3.7):

$$
d \phi_{\rho}=d x^{\sigma}\left(A^{-1}\right)_{\sigma}^{\tau}\left\{\phi_{\tau}, \phi_{\rho}\right\} \quad(\rho, \sigma, \tau=0, \ldots, m) .
$$

We may put Eqs. (3.37) in a more explicit form. Under the hypothesis (3.33), Eqs. (3.38) can be solved in terms of $p_{\rho}$ $(\rho=0,1, \ldots, m)$ :

$$
p_{\rho}-\psi_{\rho}\left(p_{\rho^{\prime}}, x^{\alpha}\right)=0 \quad\left(\rho^{\prime}=m+1, \ldots, n\right),
$$

so that the equations

$$
\phi_{\rho}\left(x^{\alpha}, p_{\rho^{\prime}}, p_{\rho}=\psi_{\rho}\left(p_{\rho^{\prime}}, x^{\alpha}\right)\right)=0
$$

are identities in $x^{\alpha}$ and $p_{\rho}$. On the basis of the same argument applied to Eq. (3.17), we get the following weak equations:

$$
\left\{\begin{array}{l}
\frac{\partial \phi_{\rho}}{\partial x^{\alpha}} \approx-A_{\rho}^{\sigma}\left\{p_{\alpha}, p_{\sigma}-\psi_{\sigma}\right\}, \\
\frac{\partial \phi_{\rho}}{\partial p_{\alpha}} \approx A_{\rho}^{\cdot \sigma}\left\{x^{\alpha}, p_{\sigma}-\psi_{\sigma}\right\},
\end{array}\right.
$$

so that the characteristic system (3.37) can be rewritten as

$$
\begin{aligned}
& d x^{\alpha}=\left\{x^{\alpha}, p_{\rho}-\psi_{\rho}\right\} d x^{\rho}, \\
& d p_{\alpha}=\left\{p_{\alpha}, p_{\rho}-\psi_{\rho}\right\} d x^{\rho}, \\
& d S=p_{\alpha}\left\{x^{\alpha}, p_{\rho}-\psi_{\rho}\right\} d x^{\rho}, \\
& \phi_{\rho}=0 .
\end{aligned}
$$


We already know from Eqs. (3.8) that this system is integrable. This can easily be checked with the use of the Jacobi identity (here we are speaking of local integrability) and remembering that the constraints in the form $p_{\rho}-\psi_{\rho}=0$ are in involution among themselves.

The equation for $S$, after integration of the characteristic equations, will give the Hamilton-Jacobi function evaluated along the characteristic surfaces (which are $m+1$ dimensional). From this it is possible (even when second-class constraints are absent) to recover the function $S=S(x)$ by eliminating half of the integration constants in favor of an equal number of coordinates. We will not discuss this point but rather the inverse procedure which consists of finding from $S$ the solutions of the equations of motion (characteristic equations).

Indeed the knowledge of a complete integral of the Hamilton-Jacobi equations $S=\phi\left(x^{\alpha}, c_{k}\right)+c$ gives a solution of the equations of motion (3.40) (characteristic manifold). As is well known from analytical mechanics, a complete integral can be used in the following way: from

$$
\begin{array}{ll}
S-\phi\left(x^{\alpha}, c_{k}\right)-c=0 & (k=m+1, \ldots, n), \\
\frac{\partial \phi\left(x^{\alpha}, c_{k}\right)}{\partial c_{h}}-b^{h}=0 & (h=m+1, \ldots, n),
\end{array}
$$

where $b^{h}$ are new $n-m$ constants, and adding Eqs. (3.15) to these,

$$
p_{\alpha}-f_{\alpha}\left(x^{\beta}, c_{k}\right)=0,
$$

where $f_{\alpha}(x, c)=\partial \phi(x, c) / \partial x^{\alpha}$, we get a submanifold of $R^{2 n+3}$ with dimension equal to $m+1$ for any given value of the $2(n-m)+1$ constants $c_{k}, b^{k}$, and $c$. If we can show that the characteristic vector fields (3.3) are tangent to each of these manifolds, that is, for any choice of the constants $c_{k}, b^{k}$, and $c$, we will have demonstrated that these are the characteristic manifolds.

This is easily checked. In fact we have

$$
\begin{aligned}
v_{\rho}(S-\phi(x, c)-c) & =-\left\{\phi_{\rho}, S-\phi(x, c)-c\right\}_{n h} \\
& =\frac{\partial \phi_{\rho}}{\partial p_{\alpha}}\left(p_{\alpha}-\frac{\partial \phi}{\partial x^{\alpha}}\right)=0, \\
v_{\rho}\left(\frac{\partial \phi(x, c)}{\partial c_{h}}-b^{h}\right) & =-\frac{\partial \phi_{\rho}}{\partial p_{\alpha}} \frac{\partial^{2} \phi}{\partial c_{h} \partial x^{\alpha}} \\
& =-\frac{\partial}{\partial c_{h}} \phi_{\rho}\left(x^{\alpha}, p_{\alpha}=f_{\alpha}(x, c)\right)=0,
\end{aligned}
$$

since the equations $\phi_{\rho}=0$ are satisfied identically by $p_{\alpha}=f_{\alpha}(x, c)$, and finally

$$
\begin{aligned}
v_{\rho}\left(p_{\alpha}-\frac{\partial \phi}{\partial x^{\alpha}}\right) & =-\left(\frac{\partial \phi_{\rho}}{\partial x^{\alpha}}+\frac{\partial \phi_{\rho}}{\partial p_{\beta}} \frac{\partial^{2} \phi}{\partial x^{\beta} \partial x^{\alpha}}\right) \\
& =-\frac{\partial}{\partial x^{\alpha}} \phi_{\rho}\left(x^{\alpha}, p_{\alpha}=f_{\alpha}(x, c)\right)=0
\end{aligned}
$$

with the same argument.

Let us observe that the argument is the same as in standard Hamilton-Jacobi theory. This can be understood by observing that a constrained system is nothing more than a particular case of a classical system where a certain number of constants of motion, the constants $c_{\rho}$ of Eq. (3.14), are required to be zero, instead of being arbitrary constants. For this reason, the dimension of the characteristic manifold is not $2(n+1)-1$, but $2(n+1)-(m+1)$.

\section{THE CHARACTERISTIC SYSTEM FOR THE CASE $\mathbf{R} \neq \mathbf{0}$}

When $r \neq 0$, due to condition (2.10), the set of constraints cannot be interpreted as a set of PDE in the unknown $S$. Strictly speaking, as we pointed out in Sec. II, a Hamilton-Jacobi function does not exist (it can, however, exist in some reduced space; see below).

Nevertheless, the characteristic system exists and is integrable, as we will see in this section. Moreover, some use can again be made of the Hamilton-Jacobi approach developed in the last section.

Let us first discuss the characteristic system, which should be constructed by starting from the same equations (2.17), (2.18), and (2.19), and the condition (2.20).

Since the rank of the matrix $\left\|\left\{\phi_{\rho}, \phi_{\sigma}\right\}\right\|$ is now $r \neq 0$, there will exist a minor of rank $r$ different from zero, which we will assume to be formed with the last $r$ rows and columns of $\left\|\left\{\phi_{\rho}, \phi_{\sigma}\right\}\right\|$ :

$$
\left\{\phi_{\mu^{\prime}}, \phi_{v^{\prime}}\right\}=C_{\mu^{\prime} v^{\prime}} \quad\left(\mu^{\prime}, v^{\prime}=m-r+1, \ldots, m\right),
$$

with

$$
\left|C_{\mu^{\prime} v^{\prime}}\right| \neq 0,
$$

where $r$ must be even, due to the antisymmetry of the matrix $\left\|\left\{\phi_{\rho}, \phi_{\sigma}\right\}\right\| ;$ we will put $r=2 s$.

In the case $r<m+1$, Eqs. (2.20) will give $r$ of $\lambda^{\sigma}$ in terms of the remaining $m-r+1$ :

$$
\begin{aligned}
& \lambda^{\mu^{\prime}} \approx-\left(C^{-1} \mu^{\mu^{\prime} v^{\prime}}\left\{\phi_{v^{\prime}}, \phi_{\mu}\right\} \lambda^{\mu}\right. \\
& \left(\mu^{\prime} v^{\prime}=m-r+1, \ldots, m, \mu, v=0, \ldots, m-r=f\right)
\end{aligned}
$$

or

$$
\lambda^{\rho} \approx\left(\delta_{\mu}^{\rho}-\delta_{\mu^{\prime}}^{\rho}\left(C^{-1}\right)^{\mu^{\prime} \gamma^{\prime}}\left\{\phi_{v^{\prime}}, \phi_{\mu}\right\}\right) \lambda^{\mu} \quad(\rho=0, \ldots, m),
$$

which for $\rho=v$ is an identity.

The case $r=m+1$, which is possible only when $m+1$ is even, will be discussed at the end of this section.

If we substitute in Eqs. (2.17), we get

$$
\begin{aligned}
& v^{\alpha}=\lambda^{\mu}\left(\delta_{\mu}^{\rho}-\delta_{\mu^{\prime}}^{\rho}\left(C^{-1}\right)^{\mu^{\prime} v^{\prime}}\left\{\phi_{v^{\prime}}, \phi_{\mu}\right\}\right)\left\{x^{\alpha}, \phi_{\rho}\right\}, \\
& u_{\alpha}=\lambda^{\mu}\left(\delta_{\mu}^{\rho}-\delta_{\mu^{\prime}}^{\rho}\left(C^{-1}\right)^{\mu^{\prime} v^{\prime}}\left\{\phi_{v^{\prime}}, \phi_{\mu}\right\}\right)\left\{p_{\alpha}, \phi_{\rho}\right\},
\end{aligned}
$$

which besides Eqs. (2.18) give the new set of characteristic vector fields:

$$
v_{\mu}=-\left\{\phi_{\mu}, \cdot\right\}_{n h}^{*},
$$

where we have defined the nonhomogeneous Dirac bracket:

$$
\{f, g\}_{n h}^{*}=\{f, g\}_{n h}-\left\{f, \phi_{\mu^{\prime}}\right\}_{n h}\left(C^{-1}\right)^{\mu^{\prime} v^{\prime}}\left\{\phi_{v^{\prime}}, g\right\}_{n h} \cdot(4.6
$$

The dimension of the space $\widetilde{Q}$ is now $m-r+1$.

The system of equations

$$
v_{\mu}(g)=-\left\{\phi_{\mu}, g\right\}_{n h}^{*}=0,
$$

associated with the set of characteristic vector fields (4.5), is completely integrable on the surface (2.6). This can be verified, as in Sec. III, by using a generalization of the Mayer 
identity to Dirac brackets. By a very long computation it can be verified that

$$
\left\{f_{1},\left\{f_{2}, f_{3}\right\}_{n h}^{*}\right\}_{n h}^{*}+\frac{\partial f_{1}}{\partial S}\left\{f_{2}, f_{3}\right\}_{n h}^{*}+\text { cyclic }=0 .
$$

Let us observe that the characteristic vectors $(4.5)$ can be rewritten in an equivalent way as

$$
v_{\mu}=-\left\{\phi_{\mu}^{*}, \cdot\right\}_{n h},
$$

where $\phi_{\mu}^{*}$ are the "starred" variables defined in (A1). They are first class and satisfy

$$
\begin{aligned}
& \left\{\phi_{\mu}^{*}, \phi_{v}^{*}\right\} \equiv O\left(\phi_{\mu}^{*}\right)+O\left(\phi_{\mu^{\prime}}^{2}\right) \\
& \left(\lambda, \mu, v=0, \ldots, m-r, \mu^{\prime}, v^{\prime}=m-r+1, \ldots, m\right) .
\end{aligned}
$$

At this point we could try to look for an involutory system starting from the set $\phi_{\mu}^{*}$, in analogy to the method followed in Sec. III. But it is easily realized that, in the presence of second-class constraints, one cannot get an analogous set of $n+1$ functions (or better $n+2$, taking into account the function $S$ as we did in Sec. III). Indeed, the system (4.7) with $v_{\mu}$ in the form (4.9) has $2 n+3-(f+1)$ solutions, of which $f+1+2 s$ are already known [the $2 s$ second-class functions $\phi_{\mu^{\prime}}$ satisfy by construction the system (4.7)]. Therefore, by selecting from among these solutions a set of functions to be added to $\phi_{\mu^{*}}^{*}: G_{1}(x, p, S), G_{2}(x, p, S), \ldots$, we can form from these the corresponding starred quantities, $G_{1}^{*}, \mathrm{G}_{2}^{*}, \cdots$, in order to preserve the solutions we already know.

In this way we get the maximum set

$$
G_{1}^{*}, G_{2}^{*}, \ldots, G_{n+1-(f+s)}^{*}
$$

of functions in weak involution with respect to the nonhomogeneous Poisson bracket.

The characteristic system can now be constructed starting from this definition of the characteristic vector fields, by eliminating $\lambda^{\mu}$ from the following expression of the components of a general characteristic vector field:

$$
\begin{aligned}
& x^{\alpha}=\lambda^{\mu}\left\{x^{\alpha}, \phi_{\mu}^{*}\right\}, \\
& u_{d}=\lambda^{\mu}\left\{p_{\alpha}, \phi_{\mu}^{*}\right\}, \\
& v^{n+1}=p_{\alpha} v^{\alpha} .
\end{aligned}
$$

If we put

$$
A_{\mu}^{v}=\left\{x^{v}, \phi_{\mu}^{*}\right\}
$$

and if we make the usual assumption that

$$
\left|\frac{\partial \phi_{\mu}^{*}}{\partial p_{v}}\right| \neq 0
$$

we get

$$
\begin{aligned}
& v^{\alpha}-v^{v}\left(A^{-1}\right)_{v}^{\mu}\left\{x^{\alpha}, \phi_{\mu}^{*}\right\}=0, \\
& u_{\alpha}-v^{v}\left(A^{-1}\right)_{\nu}^{\mu}\left\{p_{\alpha}, \phi_{\mu}^{*}\right\}=0, \\
& v^{n+1}-p_{\alpha} v^{\alpha}=0 .
\end{aligned}
$$

With the substitutions $v^{\alpha} \rightarrow d x^{\alpha}, u_{\alpha} \rightarrow d p_{\alpha}$, and $v^{n+1} \rightarrow d S$ in (4.13), we get the following differential forms:

$$
\begin{aligned}
& \eta^{\alpha}=d x^{\alpha}-d x^{\nu}\left(A^{-1}\right)_{\nu}^{\mu}\left\{x^{\alpha}, \phi_{\mu}^{*}\right\}, \\
& \zeta_{\alpha}=d p_{\alpha}-d x^{\nu}\left(A^{-1}\right)_{\nu}^{\mu}\left\{p_{\alpha}, \phi_{\mu}^{*}\right\}, \\
& \theta=d S-p_{\alpha} d x^{\alpha},
\end{aligned}
$$

and besides

$$
\begin{aligned}
& d \phi_{\mu}^{*}=\frac{\partial \phi_{\mu}^{*}}{\partial x^{\alpha}} d x^{\alpha}+\frac{\partial \phi_{\mu}^{*}}{\partial p_{\alpha}} d p_{\alpha}, \\
& d \phi_{\mu^{\prime}}=\frac{\partial \phi_{\mu^{\prime}}}{\partial x^{\alpha}} d x^{\alpha}+\frac{\partial \phi_{\mu^{\prime}}}{\partial p_{\alpha}} d p_{\alpha}
\end{aligned}
$$

The characteristic equations are now obtained by weakly putting these forms to zero; however, it is easily recognized that the last two forms, $d \phi_{\mu}^{*}$ and $d \phi_{\mu^{\prime}}$, are not independent from the others. Indeed we have

$$
\begin{aligned}
d \phi_{\mu}^{*}= & \frac{\partial \phi_{\mu}^{*}}{\partial x^{\alpha}}\left(\eta^{\alpha}+d x^{\nu}\left(A^{-1}\right)_{\nu}^{\lambda}\left\{x^{\alpha}, \phi_{\lambda}^{*}\right\}\right) \\
& +\frac{\partial \phi_{\mu}^{*}}{\partial p_{\alpha}}\left(\zeta_{\alpha}+d x^{\nu}\left(A^{-1}\right)_{\nu}^{\lambda}\left\{p_{\alpha}, \phi_{\lambda}^{*}\right\}\right) \\
= & \frac{\partial \phi_{\mu}^{*}}{\partial x^{\alpha}} \eta^{\alpha}+\frac{\partial \phi_{\mu}^{*}}{\partial p_{\alpha}} \zeta_{\alpha}+d x^{\nu}\left(A^{-1}\right)_{\nu}^{\cdot \lambda}\left\{\phi_{\mu}^{*}, \phi_{\lambda}^{*}\right\} \\
\approx & \frac{\partial \phi_{\mu}^{*}}{\partial x^{\alpha}} \eta^{\alpha}+\frac{\partial \phi_{\mu}^{*}}{\partial p_{\alpha}} \zeta_{\alpha} .
\end{aligned}
$$

In conclusion we have a set of $(2 n+3)-(m-2 s+1)$ independent differential forms

$\left[\operatorname{dim} Q^{*}=(2 n+3)-(m-2 s+1)\right]$, and the characteristic system is given by

$$
\begin{aligned}
& d x^{\alpha}=d x^{\nu}\left(A^{-1}\right)_{\nu}^{\mu}\left\{x^{\alpha}, \phi_{\mu}^{*}\right\}, \\
& d p_{\alpha}=d x^{\nu}\left(A^{-1}\right)_{\nu}^{\mu}\left\{p_{\alpha}, \phi_{\mu}^{*}\right\}, \\
& d S=p_{\alpha} d x^{\alpha}, \\
& \phi_{\mu}^{*}=0, \quad \phi_{\mu^{\prime}}=0 .
\end{aligned}
$$

Following the same discussion as in Sec. III, we have the characteristic manifold parametrized by

$2(n-m+s)+1$ arbitrary constants, where one of these is an unessential additive constant for $S$.

We can put the characteristic equations in a form analogous to that of Eqs. (3.40), if we solve the equations $\phi_{\mu}^{*}=0$ in terms of $p_{\mu}$,

$$
p_{\mu}=\psi_{\mu}\left(x^{\alpha}, p_{\mu^{\prime}}\right)
$$

$\left(\mu=0,1, \ldots, m-2 s, \mu^{\prime}=m-2 s+1, \ldots, n\right)$.

Following the same arguments, we now get

$$
\left\{p_{\mu}-\bar{\psi}_{\mu}, p_{v}-\bar{\psi}_{v}\right\}=O\left(\phi_{\mu^{\prime}}^{2}\right)
$$

where we have used Eq. (4.10), whereas, in the case of firstclass constraints only, we would have gotten zero. It is easily verified that

$$
\begin{aligned}
& \frac{\partial \phi_{\mu}^{*}}{\partial x^{\alpha}}=-A_{\mu}^{v}\left\{p_{\alpha}, p_{v}-\bar{\psi}_{v}\right\}, \\
& \frac{\partial \phi_{\mu}^{*}}{\partial p_{\alpha}}=+A_{\mu}^{\nu}\left\{x^{\alpha}, p_{v}-\bar{\psi}_{v}\right\}
\end{aligned}
$$

when (4.17) holds; so we can write the characteristic system in the following form:

$$
d x^{\alpha}=d x^{v}\left\{x^{\alpha}, p_{v}-\bar{\psi}_{v}\right\},
$$




$$
\begin{aligned}
& d p_{\alpha}=d x^{v}\left\{p_{\alpha}, p_{v}-\bar{\psi}_{v}\right\}, \\
& d S=p_{\alpha} d x^{\alpha}, \\
& \phi_{\mu}^{*}=0, \quad \phi_{\mu^{\prime}}=0 .
\end{aligned}
$$

In the form (4.20) we can more easily verify that the integrability conditions are satisfied, due to the property (4.18), using the Jacobi identity. As regards Eqs. (4.21), the same comment we made in Sec. III applies here as well. The point is rather that knowledge of the functions $S$ evaluated along the characteristics is not enough to recover a function $S$ of the coordinates $x^{\alpha}$ since, as we already know, such a function does not exist when second-class constraints are present.

When $m+1$ is even and $r=m+1$, by repeating the discussion we find $\operatorname{dim} \widetilde{Q}=0$ and the associated space $Q^{*}$ will have dimension $2 n+3$. So it will be spanned by any $(2 n+3)$-dimensional basis, which can be chosen $\left(d x^{\alpha}, d S, d p_{\alpha}\right)$.

As a result the associated Pfaff system will be

$$
\begin{aligned}
& d x^{\alpha}=0, \\
& d p_{\alpha}=0, \quad \phi_{\rho}(x, p)=0, \\
& d S=0,
\end{aligned}
$$

which agrees with Eqs. (4.16) when no first-class contraints exist. As in the previous case the Hamilton-Jacobi function $S=S\left(x^{\alpha}\right)$ does not exist.

\section{THE HAMILTON-JACOBI FUNCTION}

In the last section we saw how to get a characteristic system when second-class constraints are present, and we stressed that in such a case a function $S$ satisfying only the constraint equations (if we think of them as PDE in $S$ ) does not exist. ${ }^{18}$ So we cannot really speak of a Hamilton-Jacobi method in such cases. Nevertheless, as we will see in the present section, the theory developed in Sec. III can again be useful.

We will consider two situations: the first is when the first-class constraints form a first-class subset, that is, when the Poisson bracket between any two of them is a first-class constraint. In such a case we will say that they are in weak involution among themselves. This can always be achieved as an application of general theorems. ${ }^{16}$

In this situation let us consider this set as defining our dynamical system, neglecting for the moment the secondclass constraints. This set

$$
\phi_{\mu}(x, p)=0 \quad(\mu=0, \ldots, f)
$$

can be considered as a system of PDE in $S=S(x)$, following the theory developed in Sec. III.

Let us suppose that we have found a complete integral of the system (5.1),

$$
S=\phi\left(x^{\alpha}, c_{k}\right)+c \quad(\alpha=0, \ldots, n, k=f+1, \ldots, n),
$$

where $c_{k}$ and $c$ are $n-f+1$ arbitrary constants.

In order to find the solutions of the characteristic equations, we put, as in Eqs. $\left(3.41^{\prime}\right)$,

$$
\frac{\partial \phi(x, c)}{\partial c_{h}}-b^{h}=0,
$$

where $b^{h}$ are new $n-f$ constants. By adding to Eqs. (5.3) Eqs. (5.2) and

$$
p_{\alpha}-f_{\alpha}(x, c)=0
$$

where

$$
f_{\alpha}(x, c)=\frac{\partial \phi(x, c)}{\partial x^{\alpha}},
$$

we get $2 n+3-(f+1)$ equations for $x^{\alpha}, p_{\alpha}$, and $S$, which can be solved for $p_{\alpha}$ and $n-f x^{\alpha}$, regarding $f+1$ of the coordinates $x^{\alpha}$ as parameters. We thus obtain an integral manifold for any given value of the $2(n-f)+1$ constants $c_{k}$, $b^{k}$, and $c$, as in Sec. III (the substitution of $f$ in place of $m$ must be performed).

The solution of the original characteristic system (4.16) can be derived from this solution by adding to the solution just found the second-class constraints $\phi_{\mu^{\prime}}(x, p)=0$, which turn out to be constant and so have the meaning of some restriction on the constants $c_{k}$ and $b^{k}$. The final number of the constants will be $2(n-f)+1-2 s$.

In order to show this, let us write the characteristic vectors of the exterior differential system (5.1). These are, according to Sec. III [see Eq. (3.3)]:

$$
v_{\mu}=-\left\{\phi_{\mu}, \cdot\right\}_{n h} \text {. }
$$

The hypothesis made on $\phi_{\mu}$ now has the important consequence that $v_{\mu}$ are weakly equal to the characteristic vectors of our original system, Eq. (4.5), i.e., when they are calculated on the submanifold defined by all the constraints $\phi_{\mu}$ and $\phi_{\mu^{\prime}}$ :

$$
v_{\mu}=-\left\{\phi_{\mu}, \cdot\right\}_{n h} \approx-\left\{\phi_{\mu}, \cdot\right\}_{n h}^{*} .
$$

In fact, using the explicit expression of the Dirac brackets [see Eq. (4.6)], we have

$$
\left\{\phi_{\mu}, \cdot\right\}_{n h}^{*}=\left\{\phi_{\mu}, \cdot\right\}_{n h}-\left\{\phi_{\mu}, \phi_{\mu^{\prime}}\right\}_{n h}\left(C^{-1}\right)^{\mu^{\prime} v^{\prime}}\left\{\phi_{v^{\prime}}, \cdot\right\}_{n h},
$$

where the last term on the rhs is weakly zero when $\phi_{\mu}=0$ and $\phi_{\mu^{\prime}}=0$. This is because $\phi_{\mu}$ are assumed to be first class.

This fact has the consequence that, when all the constraints are satisfied, we have

$$
v_{\mu}\left(\phi_{\mu^{\prime}}\right) \approx 0
$$

that is, the characteristic vectors of the integral manifold we have found are also tangent to our original submanifold defined by the equations $\phi_{\mu}=0$ and $\phi_{\mu^{\prime}}=0$.

This demonstrates that the restrictions imposed on the solution $(5.3),(5.4)$ by $\phi_{\mu^{\prime}}(x, p)=0$ are indeed restrictions on the constants $c_{k}$ and $b^{k}$, and that in this way a solution of the original system can be found.

Another situation that may occur is when the constraints $\phi_{\mu}$ and $\phi_{\mu^{\prime}}$ admit a maximal subset of first-class constraints (among themselves). We know from a general theorem that this always takes place, at least locally. Indeed we know that it is always possible to locally substitute the set $\phi_{\mu}$ and $\phi_{\mu^{\prime}}$ with a new set such that $f+s+1$ of them are first class among themselves and the remaining $s$ are all second class. ${ }^{16}$

In general it is very difficult to find such a maximal subset, and doing so could be equivalent in almost all cases to completely solving the dynamics.

Nevertheless, it may turn out to be possible, or the con- 
straints may already satisfy this condition.

If this is the case, let us call this subset

$$
\psi_{\lambda}(x, p)=0 \quad(\lambda=0,1, \ldots, f+s) .
$$

The $\psi_{\lambda}$ include $\phi_{\mu}, \mu=0,1, \ldots, f$, and half of $\phi_{\mu^{\prime}}$. Equations (5.9) alone define a Hamilton-Jacobi function $S\left(x^{\alpha}\right)$. So let us for the moment put aside the remaining constraints $\phi_{\mu^{\prime}}$, $\mu^{\prime}=f+s+1, \ldots, f+2 s+1$, which we will call

$$
\begin{aligned}
& \phi_{\mu^{\prime}}(x, p)=\phi_{f+s+l}(x, p) \\
& \left(\mu^{\prime}=f+s+1, \ldots, f+2 s, l=1, \ldots, s\right) .
\end{aligned}
$$

Let us suppose we have found a complete integral for the set of first-class (5.9):

$$
S=\Psi\left(x, c_{k}\right)+c[k=1, \ldots, n-(f+s)],
$$

where $c_{k}$ and $c$ are $n-f-s+1$ arbitrary constants.

We want to show that a solution of our original system (4.16) can be obtained by imposing the conditions $(5.10)$ on the characteristic surface obtained from (5.11) by means of the equations

$$
\frac{\partial \Psi(x, c)}{\partial c_{h}}-b^{h}=0
$$

where $b^{h}$ are new $n-f-s$ constants, and

$$
p_{\alpha}-f_{\alpha}\left(x^{\beta}, c_{k}\right)=0
$$

with

$$
f_{\alpha}\left(x^{\beta}, c_{k}\right)=\frac{\partial \Psi(x, c)}{\partial x^{\alpha}} .
$$

The solution given by these $2 n+3-(f+s+1)$ equations can be expressed by solving $p_{\alpha}$ and $n-(f+s)$ of $x^{\alpha}$ in terms of the remaining $f+s+1$ coordinates regarded as parameters and the constants $c_{k}$ and $b^{k}$. The integral manifold so obtained has dimension $f+s+1$ for any given value of the $2(n-f-s)+1$ constants $c_{k}, b^{k}$, and $c$ in $\mathbb{R}^{2 n+3}$.

In order to show this, let us write the characteristic vectors of the system (5.9), which are now

$$
v_{\lambda}=-\left\{\psi_{\lambda}, \cdot\right\}_{n h} \quad(\lambda=0, \ldots, f+s) .
$$

The situation is now quite different from the previous case. The analogous equation (5.7) does not hold any more.

If we require the integral manifold we have found to be an integral manifold of the system $\phi_{f+s+l}(x, p)=0$ as well, its dimensions will diminish from $f+s+1$ to $f+1$. In order to get this result, we must require that the characteristic space spanned by the vectors $v_{\lambda}$ of Eq. (5.14) be restricted by the requirement

$$
v\left(\phi_{f+s+l}\right) \approx 0 \quad(l=1, \ldots, s),
$$

where $v$ is a generic vector given by

$$
v=\lambda^{\lambda} v_{\lambda} \quad(\lambda=0, \ldots, f+s) .
$$

Equations (5.15) will give

$$
\lambda^{\lambda} v_{\lambda}\left(\phi_{f+s+l}\right)=-\lambda^{\lambda}\left\{\psi_{\lambda}, \phi_{f+s+l}\right\}=0 .
$$

Since the rank of the matrix $\left\|\left\{\psi_{\lambda}, \phi_{f+s+l}\right\}\right\|$ is $s$ (in a weak sense), we can solve Eqs. (5.17) for $s$ of $\lambda^{\lambda}$ in terms of the remaining $f+1$ :

$$
\begin{aligned}
& \lambda^{f+\mu}=-\lambda^{\mu}\left\{\phi_{\mu}, \phi_{f+s+l}\right\}\left(C^{-1}\right)^{l m} \\
& (l, m=1, \ldots, s, \mu=0, \ldots, f),
\end{aligned}
$$

where

$$
C_{l m}=\left\{\phi_{f+s+l}, \phi_{f+m}\right\} .
$$

In Eqs. (5.18) we have used the notation of Sec. IV regarding the labelling of the indices.

By substituting (5.18) in (5.16) we get

$$
\begin{aligned}
v & =\lambda^{\mu}\left[v_{\mu}-\left\{\phi_{\mu}, \phi_{f+s+l}\right\}\left(C^{-1}\right)^{l m} v_{m}\right] \\
& =-\lambda^{\mu}\left[\left\{\phi_{\mu}, \cdot\right\}_{n h}-\left\{\phi_{\mu}, \phi_{f+s+l}\right\}\left(C^{-1}\right)^{l m}\left\{\phi_{f+m},\right\}_{n h}\right] .
\end{aligned}
$$

(The nonhomogeneous Poisson bracket between two constraints coincides with the usual Poisson bracket, since they do not depend on $S$.)

By adding to the lhs side of Eq. (5.19) terms which are zero when all the constraints are satisfied, we get

$$
\begin{aligned}
v \approx & -\lambda^{\mu}\left[\left\{\phi_{\mu}, \cdot\right\}_{n h}-\left\{\phi_{\mu}, \phi_{f+s+l}\right\}_{n h}\left(C^{-1}\right)^{l m}\left\{\phi_{f+m}, \cdot\right\}_{n h}\right. \\
& +\left\{\phi_{\mu}, \phi_{f+l}\right\}_{n h}\left(\widetilde{C}^{-1}\right\}^{I m}\left\{\phi_{f+s+m}, \cdot\right\}_{n h} \\
& -\left\{\phi_{\mu}, \phi_{f+l}\right\}_{n h}\left(C^{-1}\right)^{I m}\left\{\phi_{f+s+m}, \phi_{f+s+n}\right\}_{n h} \\
& \left.\times\left(\widetilde{C}^{-1}\right)^{n p}\left\{\phi_{f+p}, \cdot\right\}_{n h}\right]
\end{aligned}
$$

$(l, m, n, p=1, \ldots, s)$, where $\widetilde{C}_{l m}=C_{m l}$. We recognize the structure of the Dirac brackets:

$$
v \approx-\lambda^{\mu}\left\{\phi_{\mu}, \cdot\right\}_{n h}^{*} \text {. }
$$

In this way we recover the characteristic vectors (4.5):

$v_{\mu}=-\left\{\phi_{\mu},\right\}_{n h}^{*}$.

In practice, to get this result when working on the solution (5.12), (5.13), it is only necessary to impose on this solution the restriction

$$
\phi_{f+s+l}(x, p)=0,
$$

where now, contrary to the first case considered in this section, Eq. (5.22) has the meaning of a restriction on the coordinates [which in the number of $f+s+1$ can be used to parametrize the solution (5.12) and (5.13)] and not on the constants $c_{k}, b^{k}$, which in this case are in the correct number right from the start.

The two situations described are two examples of the use of the Hamilton-Jacobi approach when second-class constraints are present. The method could be extended to intermediate cases where a number of constraints between $f+1$ and $f+s+1$ are known to be a first-class subset (among themselves).

\section{PARAMETRIC FORMS OF THE CHARACTERISTIC SYSTEM}

Until now we have discussed a parameter-free approach which in our opinion is the main feature of the HamiltonJacobi theory. We wish to mention here the possibility of a multiparameter approach. We can try to reformulate Eqs. (3.37) by defining $m+1$ parameters $\omega^{\rho}$ by the equations

$$
d \omega^{\rho}=d x^{\sigma}\left(A^{-1}\right)_{\sigma}^{\rho} \quad(\rho, \sigma=0, \ldots, m) .
$$

If this is possible the characteristic equations (3.37) become

$$
\begin{aligned}
& d x^{\alpha}=\left\{x^{\alpha}, \phi_{\rho}\right\} d \omega^{\rho}, \\
& d p_{\alpha}=\left\{p_{\alpha}, \phi_{\rho}\right\} d \omega^{\rho}, \quad \phi_{\rho}=0 . \\
& d S=p_{\alpha}\left\{x^{\alpha}, \phi_{\rho}\right\} d \omega^{\rho},
\end{aligned}
$$


If we check the integrability conditions of the Mayer system deduced from Eqs. (6.2) using the Jacobi identity, we find that these conditions boil down to requiring

$$
\left\{\phi_{\rho}, \phi_{\sigma}\right\}=O\left(\phi^{2}\right) \text {. }
$$

Thus a multiparametric formulation such as (6.2) is possible only if the constraints $\phi_{\rho}(\rho=0, \ldots, m)$ satisfy the condition (6.3). The same remains true also for the characteristic system for a set of first- and second-class constraints [Eqs.

(4.16)]: the system

$$
\begin{aligned}
& d x^{\alpha}=\left\{x^{\alpha}, \phi_{\mu}^{*}\right\} d \omega^{\mu}, \\
& d p_{\alpha}=\left\{p_{\alpha}, \phi_{\mu}^{*}\right\} d \omega^{\mu}, \\
& d S=p_{\alpha}\left\{x^{\alpha}, \phi_{\mu}^{*}\right\} d \omega^{\mu}, \\
& \phi_{\mu}^{*}=0, \quad \phi_{\mu^{\prime}}=0,
\end{aligned}
$$

is integrable only if

$$
\left\{\phi_{\mu}^{*}, \phi_{v}^{*}\right\}=O\left(\phi^{2}\right) \text {. }
$$

Besides (6.3) there is another case where one can use a multiparametric formulation. Namely, if the constraints $\phi_{\rho}$ (or $\phi_{\mu}$ ) form a closed Lie algebra under the Poisson (or Dirac) brackets, i.e.,

$$
\left\{\phi_{\rho}, \phi_{\sigma}\right\}=C_{\rho \sigma}^{\tau} \phi_{\tau},
$$

where $C_{\rho \sigma}^{r}$ denote the structure constants, then the equations

$$
\begin{aligned}
& d x^{\alpha}=\left\{x^{\alpha}, \hat{\phi}_{\rho}\right\} d \tau^{\rho}, \\
& d p_{\alpha}=\left\{p_{\alpha}, \hat{\phi}_{\rho}\right\} d \tau^{\rho}
\end{aligned}
$$

with

$$
\hat{\phi}_{\rho}=B_{\rho}^{\sigma}(\tau) \phi_{o},
$$

where the functions $B_{\rho}^{\sigma}(\tau)$ satisfy

$$
\frac{\partial}{\partial \tau^{\tau}} B_{\rho}^{\sigma}-\frac{\partial}{\partial \tau^{\rho}} B_{\tau}^{\sigma}=B_{\tau}^{\zeta} B_{\rho}^{\eta} C_{\zeta \eta}^{\sigma},
$$

are integrable. The functions $B_{\rho}^{\sigma}(\tau)$ are determined entirely by the structure constants [see Ref. 19]. In fact it turns out that

$$
\begin{aligned}
\frac{\partial^{2} x^{\alpha}}{\partial \tau^{\sigma} \partial \tau^{\rho}} & -\frac{\partial^{2} x^{\alpha}}{\partial \tau^{\rho} \partial \tau^{\sigma}} \\
= & \left\{\left\{x^{\alpha}, \hat{\phi}_{\rho}\right\}, \hat{\phi}_{\sigma}\right\}+\left\{x^{\alpha}, \phi_{\tau}\right\} \frac{\partial B_{\rho}^{\tau}}{\partial \tau^{\sigma}}-(\rho \leftrightarrow \sigma) \\
= & -B_{\rho}^{\eta} B_{\sigma}^{\zeta} C_{\eta \xi}^{\tau}\left\{\phi_{\tau}, x^{\alpha}\right\} \\
& +\left\{x^{\alpha}, \phi_{\tau}\right\}\left(\frac{\partial B_{\rho}^{\tau}}{\partial \tau^{\sigma}}-\frac{\partial B_{\sigma}^{\tau}}{\partial \tau^{\rho}}\right)=0
\end{aligned}
$$

and analogously for $p_{\alpha}$. The calculation goes in the same way even when second-class constraints are present.

Finally, let us consider a one-parameter formulation, by recovering the equations of motion derived by Dirac. This can be achieved by choosing a particular vector field of the characteristic "manifold"

$$
v=-\lambda^{\mu}\left\{\phi_{\mu},\right\}^{*} \quad(\mu=0, \ldots, m-r) .
$$

The corresponding Pfaff system is given by

$$
d x^{\alpha}=\lambda^{\mu}\left\{x^{\alpha}, \phi_{\mu}\right\}^{*} d \tau,
$$

$$
\begin{aligned}
& d p_{\alpha}=\lambda^{\mu}\left\{p_{\alpha}, \phi_{\mu}\right\}^{*} d \tau, \\
& (\alpha=0, \ldots, n, \mu=0, \ldots, m-r),
\end{aligned}
$$

and

$$
d S=\lambda^{\mu} p_{\alpha}\left\{x^{\alpha}, \phi_{\mu}\right\}^{*} d \tau, \quad \phi_{\mu}^{*}=0, \phi_{\mu^{\prime}}=0,
$$

where $\{,\}^{*}$ is the usual Dirac bracket.

Let us conclude this section by considering the particular case where

$$
\begin{aligned}
& \phi_{\mu}=\left\{\begin{array}{l}
\phi_{0}=p_{0}+H_{i}\left(x^{i}, p_{i}\right), \\
\phi_{A}=\phi_{A}\left(x^{i}, p_{i}\right),
\end{array}\right. \\
& (i=1, \ldots, n, A=1, \ldots, m-r) ;
\end{aligned}
$$

Eqs. (6.11) become the equations of motion for a constrained system with a nonvanishing canonical Hamiltonian (for simplicity we will choose $\lambda_{0}=1$ ):

$$
\begin{aligned}
& d x^{i}=\left\{x^{i}, H_{c}+\lambda^{A} \phi_{A}\right\}^{*} d \tau, \\
& d p_{i}=\left\{p_{i}, H_{\mathrm{c}}+\lambda^{A} \phi_{A}\right\}^{*} d \tau, \\
& d x^{0}=d \tau, \quad \phi_{A}=0, \\
& d p_{0}=0, \quad \phi_{\mu^{\prime}}=0,
\end{aligned}
$$

where we made use of the fact that $\phi_{A}$ and the second-class constraints $\phi_{\mu^{\prime}}$ do not depend on $x^{0}$ and $p_{0}$.

\section{APPENDIX A}

When first- and second-class constraints are present, that is, when the rank $r$ introduced in Sec. II is different from zero and $<m+1$, the problem of classifying the constraints $\phi_{\rho}$ into first class and second class can be solved in the following way.

We can start with the choice of a minor of maximum rank in the matrix $\left\|\left\{\phi_{\rho}, \phi_{\sigma}\right\}\right\|$. Since it is always possible to choose such a minor as a principal-hence antisymmetricminor, let us suppose it to be $\left\|\left\{\phi_{\mu^{\prime}}, \phi_{v^{\prime}}\right\}\right\|$, where $\mu^{\prime}$, $v^{\prime}=m-r+1, \ldots, m$. The remaining functions $\phi_{\mu}$ $(\mu=0,1, \ldots, m-r)$ are in general not first class, but from them we can construct a set of first-class constraints. To this end, we can observe that any row of the matrix $\left\|\left\{\phi_{\rho}, \phi_{\sigma}\right\}\right\|$ not belonging to the chosen minor must be a linear combination of the last $r$ rows. Explicitly this means

$$
\left\{\phi_{\mu}, \phi_{\mu^{\prime}}\right\} \approx A_{\mu v^{\prime}}\left\{\phi_{v^{\prime}}, \phi_{\mu^{\prime}}\right\},
$$

which can be equivalently written

$$
\left\{\phi_{\mu}-A_{\mu \nu^{\prime}} \phi_{v^{\prime}}, \phi_{\mu^{\prime}}\right\} \approx 0 \text {. }
$$

From this we see that we can define ${ }^{3}$

$$
\phi_{\mu}^{*}=\phi_{\mu}-\left\{\phi_{\mu}, \phi_{\mu^{\prime}}\right\}\left(C^{-1}\right)^{\mu^{\prime} v^{\prime}} \phi_{v^{\prime}},
$$

which have the property $\left\{\phi_{\mu}^{*}, \phi_{\mu^{\prime}}\right\} \approx 0$, with

$$
C_{\mu^{\prime} v^{\prime}}=\left\{\phi_{\mu^{\prime}}, \phi_{v^{\prime}}\right\} \text {. }
$$

We may now show that $\phi_{\mu}^{*}$ are first-class objects. Indeed let us choose $\phi_{\mu}^{*}$ and $\phi_{\mu^{\prime}}$ as a new set of constraints.

We can verify that, by doing so, the rank $r$ does not change. In fact, if we put

$$
\phi_{\mu}^{\prime}=\phi_{\mu}^{*}, \quad \phi_{\mu^{\prime}}^{\prime}=\phi_{\mu^{\prime}},
$$

we have 


$$
\phi_{\rho}^{\prime}=B_{\rho \sigma} \phi_{0},
$$

where the matrix $B$ has rank $=m+1$, since $\operatorname{det} B=1$.

Owing to this, the rank of the matrix

$$
\left\{\phi_{\rho}^{\prime}, \phi_{\sigma}^{\prime}\right\} \approx B_{\rho \lambda} B_{\sigma \tau}\left\{\phi_{\lambda}, \phi_{\tau}\right\}
$$

is again $r$. Then we necessarily have $\left\{\phi_{\mu}^{*}, \phi_{v}^{*}\right\} \approx 0$.

\section{APPENDIX B}

Under the hypotheses already postulated in Sec. II, that is, that the functions $\phi_{\rho}$ are differentiable and that the rank of $x \rightarrow\left(\phi_{0}(x), \ldots, \phi_{m}(x)\right)$ is equal to $m+1$ for every $x$ belonging to the subset $V$ of the manifold $X$ (in our case $X=\mathbb{R}^{2 n+3}$ ), defined by $\phi_{\rho}(x)=0$, the subset $V$ is a submanifold of $X$ of dimension $N-m(N=\operatorname{dim} X)($ Ref. 13, p. 228).

If $j$ denotes the inclusion mapping

$$
j: V \rightarrow X \text {, }
$$

then the pullback $j^{*}$ and the differential mapping $j_{*}$ allow us to relate structures defined on $V$ and on $X$. For instance, if $f$ is a function $f: X \rightarrow \mathbb{R}$, then

$$
j^{*} f: V \rightarrow \mathbb{R},
$$

where

$$
\left(j^{*} f\right)(x)=f(x), \quad \forall x \in V .
$$

It is convenient to introduce the following notation: If

$$
f(x)=0, \quad \omega_{x}=0, \quad \forall x \in V,
$$

where $\omega_{x}$ is a differential form on $X$, we will write

$$
f \approx 0, \omega \approx 0,
$$

and we will say that $f, \omega$ are weakly equal to zero.

Note that $f \approx 0$ is equivalent to $j^{*} f=0$, when $f$ is a 0 form, but this is no longer true for a form of arbitrary degree.

In fact, it is possible to prove that if $\alpha \in \Lambda\left(T^{*}(X)\right)$ is a differential form of degree $r, 0<r \leqslant N$, such that

$$
j^{*} \alpha=0,
$$

then

$$
\alpha \approx \sum_{\rho} a^{\rho} \wedge d \phi_{\rho},
$$

where $a^{\rho}$ is a differential form of degree $r-1$.

The proof of the previous statement lies in the following. Let $\phi_{\rho}(\rho=1, \ldots, m), \psi_{k}(k=m+1, \cdots)$ be a set of local coordinates for the manifold $X$, as is always possible under the stated hypotheses. A differential form of degree $r$ can be written as

$\alpha=\sum_{\substack{\left.(\rho) k k^{\prime}\right) \\ i=0}}^{r} \omega_{\rho_{1} \cdots \rho_{i} k_{i+1} \ldots k_{r}} d \phi_{\rho_{1}} \wedge \cdots \wedge d \phi_{\rho_{i}} \wedge d \psi_{k_{i+1}} \wedge \cdots \wedge d \psi_{k_{r}}$,

then

$$
j^{*} \alpha=0
$$

implies

$$
j^{*} \omega_{k_{1} \cdots k_{r}}=0 \Leftrightarrow \omega_{k_{1} \cdots k_{r}} \approx 0,
$$

where use is made of the fact that $j^{*}$ is an algebra homomorphism and

$$
j^{*} d \phi_{\rho}=0
$$

due to $j^{*} \phi_{\rho}=0$.

In conclusion, using Eqs. (B8) and (B11), we get

$$
\alpha \approx \sum_{\rho} a^{\rho} \wedge d \phi_{\rho}
$$

where $a^{\rho}$ is an $(r-1)$ form.

The converse statement, that Eq. (B7) implies Eq. (B6), can be easily proved.

\section{APPENDIX C}

In this appendix we want to analyze the problem posed by the presence of the 0 -forms $\phi_{\rho}$ in the exterior differential system $A$.

Stated more precisely, let us consider an exterior differential system $A$ in the manifold $X$ (in our case $\mathbf{R}^{2 n+3}$ ):

$$
A= \begin{cases}\phi_{\rho}=0 & (\rho=0, \ldots, m), \\ \omega^{\alpha}=0 & (\alpha=1, \ldots, a),\end{cases}
$$

where $\omega^{\alpha}$ are differential forms of degree greater than zero.

An integral manifold of $A$ is a pair $(M, f)$, where $M$ is a submanifold $X$ and $f$ a differentiable mapping,

$$
f: M \rightarrow X,
$$

such that

$$
\left\{\begin{array}{l}
f^{*} \phi_{\rho}=0 \\
f^{*} \omega^{\alpha}=0
\end{array}\right.
$$

Let $g$ be the mapping

$$
g: M \rightarrow V
$$

and $j$ be the inclusion mapping (B1)

$$
j: V \rightarrow X
$$

so that

$$
f=j \circ g \text {. }
$$

We know that $g$ too is a differentiable mapping. ${ }^{20}$

Now, finding an integral manifold $(M, f)$ of $A$ is equivalent to finding an integral manifold $(M, g)$ of the following system in $V$ :

$$
B=\left\{j^{*} \omega^{\alpha}=0\right\} \text {. }
$$

Indeed, if $(M, f)$ is an integral manifold of $A$ in $X$, from (C2), (C3) we get

$$
f^{*} \omega^{\alpha}=g^{*}\left(j^{*} \omega^{\alpha}\right)=0,
$$

so $(M, g)$ is an integral manifold of $B$. Conversely, if $(M, g)$ is an integral manifold of $B$, this means

$$
g^{*}\left(j^{*} \omega^{\alpha}\right)=0,
$$

that is, $f^{*} \omega^{\alpha}=0$. So the second set of Eqs. (C2) is satisfied.

On the other hand, from the definition of $V$ we get $j^{*} \phi_{\rho}=0$, which implies $g^{*}\left(j^{*} \phi_{\rho}\right)=f^{*}\left(\phi_{\rho}\right)=0$, so the system $(\mathrm{C} 2)$ is satisfied, and the pair $(M, j \circ g)$ is an integral manifold of $A$ in $X$.

As a first application of this result, let us now consider the problem of determining the characteristic vectors when 0 -forms are present. If $\bar{A}$,

$$
\bar{A}= \begin{cases}\phi_{\rho}=0, & d \phi_{\rho}=0, \\ \omega^{\alpha}=0, & d \omega^{\alpha}=0,\end{cases}
$$

and $\bar{B}$, 


$$
\bar{B}=\left\{\begin{array}{l}
j^{*} \omega^{\alpha}=0, \\
d\left(j^{*} \omega^{\alpha}\right)=j^{*} d \omega^{\alpha}=0,
\end{array}\right.
$$

are the closures of $A$ and $B$, then the differential part of $\bar{A}$ : $d \phi_{\rho}, \omega^{\alpha}, d \omega^{\alpha}$, generates the ideal $I_{A}$,

$I_{A}=\left\{\omega=\sum_{\alpha} \xi_{\alpha} \wedge \omega^{\alpha}+\sum_{\alpha} \eta_{\alpha} \wedge d \omega^{\alpha}+\sum_{\rho} \zeta^{\rho} \wedge d \phi_{\rho}\right\}$,

while $\bar{B}$ generates the ideal $I_{B}$,

$$
I_{B}=\left\{\widetilde{\omega}=\sum_{\alpha} \tilde{\xi}_{\alpha} \wedge j^{*} \omega^{\alpha}+\sum_{\alpha} \tilde{\eta}_{\alpha} \wedge j^{*} d \omega^{\alpha}\right\},
$$

where $\xi_{\alpha}, \eta_{\alpha}$, and $\xi^{\rho}$ are arbitrary forms on $X$ and $\tilde{\xi}_{\alpha}, \tilde{\eta}_{\alpha}$ are arbitrary forms on $V$.

From (C7) and (C8) we have

$$
I_{B}=j^{*} I_{A} \text {. }
$$

Let us observe that Eq. (C9) implies that the pullback of a form of $I_{A}$ belongs to $I_{B}$; we may ask whether other forms $\alpha$ belonging to $\wedge\left(T^{*}(X)\right)$ exist such that $j^{*} \alpha \in I_{B}$. We may in fact prove the following statement:

$$
j^{*} \alpha \in I_{B} \Leftrightarrow \alpha \subseteq I_{A},
$$

where the notation

$$
\alpha \in I_{A}
$$

means $\omega_{x} \in\left(I_{A}\right)_{x}$ for any $x \in V$ (we will say that the differential form $\alpha$ on $X$ weakly belongs to $I_{A}$ ).

In fact, if $j^{*} \alpha \in I_{B}$, using Eq. (C9), which tells us that a form belonging to $I_{B}$ can be written as the pullback of some form of $I_{A}$, we may write

$$
j^{*} \alpha=j^{*}\left(\sum_{\alpha} \xi_{\alpha} \wedge \omega^{\alpha}+\sum_{\alpha} \eta_{\alpha} \wedge d \omega^{\alpha}+\sum_{\rho} \zeta^{\rho} \wedge d \phi_{\rho}\right),
$$

where the term in brackets is an element of $I_{A}$. Using the fact that $j$ is an algebra homomorphism, we have

$$
j^{*} \alpha=\sum_{\alpha} j^{*} \xi_{\alpha} \wedge j^{*} \omega^{\alpha}+\sum_{\alpha} j^{*} \eta_{\alpha} \wedge j^{*} d \omega^{\alpha},
$$

from which we get

$$
j^{*}\left(\alpha-\sum_{\alpha} \xi_{\alpha} \wedge \omega^{\alpha}-\sum_{\alpha} \eta_{\alpha} \wedge d \omega^{\alpha}\right)=0 .
$$

Thus, using (B7), we have

$$
\alpha \approx \sum_{\alpha} \xi_{\alpha} \wedge \omega^{\alpha}+\sum_{\alpha} \eta_{\alpha} \wedge d \omega^{\alpha}+\sum_{\rho} \zeta^{\rho} \wedge d \phi_{\rho},
$$

that is $\alpha \in I_{A}$. The converse is easily proved in an analogous way.

From the results $(\mathrm{C} 10)$ we see that we can work in the space $X$ by considering forms which weakly belong to the ideal $I_{A}$.

The characteristic system is constructed by starting from the set of characteristic vector fields, which should be obtained from the condition

$$
i_{v}\left(I_{B}\right) \subset I_{B}, \quad v \in T(V) \text {. }
$$

We now want to demonstrate that this is equivalent to analyzing the consequences of the condition

$$
i_{\bar{v}}\left(I_{A}\right) \subset I_{A}, \quad \bar{v} \in T(X) .
$$

Indeed, if $v$ is a characteristic vector field of $B$, i.e., such that $i_{v}\left(I_{B}\right) \subset I_{B}$, since $I_{B}=j^{*} I_{A}$, this means

$$
i_{v}\left(j^{*} I_{A}\right) \subset j^{*} I_{A}
$$

or

$$
j^{*} i_{j_{*} v}\left(I_{A}\right) \subset j^{*} I_{A}
$$

Let us consider an extension $\bar{v}$ and $v$, that is a vector field on $X$ such that $\bar{v}_{x}=\left(j_{*} v\right)_{x}$ for every $x \in j V$; thus $\bar{v}$ will satisfy

$$
i_{\bar{v}} d \phi_{\rho}=0 .
$$

From Eq. (C18) we get

$$
j^{*} i_{\bar{v}}\left(I_{A}\right) \subset I_{B},
$$

and finally using Eq. (C11), we can write

$$
i_{\bar{v}}\left(I_{A}\right) \subset I_{A} \text {, }
$$

which is the result $(\mathrm{C} 17)$.

Let us observe that this result guarantees that $\bar{v}$ is actually an extension of $v$, since by applying it to the 1 -forms $d \phi_{\rho}$ of $I_{A}$ we get

$$
i_{\bar{v}}\left(d \phi_{\rho}\right) \approx 0
$$

inasmuch as no 0 -forms are present in $I_{A}$, and this is exactly the definition of $\bar{v}$.

As a second application let us study how the Frobenius theorem about the integrability of Pfaff systems must be stated, when zero forms are present.

Let us consider the system $A$ in the case where $\omega^{\alpha}$ are independent 1 -forms $\theta^{\alpha}$ :

$$
A= \begin{cases}\phi_{\rho}=0 & (\rho=0, \ldots, m), \\ \theta^{\alpha}=0 & (\alpha=1, \ldots, a) .\end{cases}
$$

We already know that this system has the same solutions as the system

$$
B=\left\{j^{*} \theta^{\alpha}=0\right\} .
$$

The Frobenius theorem states that the necessary and sufficient condition for the integrability of the system $B$ is

$$
d\left(j^{*} \theta^{\alpha}\right) \wedge\left(j^{*} \theta^{1}\right) \wedge \cdots \wedge\left(j^{*} \theta^{\alpha}\right)=0
$$

for any $\alpha$. From this, using the properties of the pullback, we get

$$
j^{*}\left(d \theta^{\alpha} \wedge \theta^{1} \wedge \cdots \wedge \theta^{a}\right)=0
$$

and from the result $(\mathbf{B} 7)$ we get

$$
d \theta^{\alpha} \wedge \theta^{1} \wedge \cdots \wedge \theta^{a} \approx \sum_{\rho} \lambda^{\rho} d \phi_{\rho},
$$

or

$$
d \theta^{\alpha} \wedge \theta^{1} \wedge \cdots \wedge \theta^{a} \wedge d \phi_{0} \wedge \cdots \wedge d \phi_{m} \approx 0,
$$

which is the integrability condition we were looking for.

The dual version of $(\mathrm{C} 22)$ can likewise be easily obtained. Indeed the dual form of the system $B$ is given by the system of vector fields

$$
B^{\prime}=\left\{v_{\lambda}\right\}, \quad v_{\lambda} \in T(V),
$$

where $\lambda=a+1, \ldots, n$, if $n$ is the dimension of the manifold, and $v_{\lambda}$ are defined by

$$
i_{v_{\lambda}}\left(j^{*} \theta^{\alpha}\right)=0
$$


for all values of $\lambda$ and $\alpha$. The dual form of the Frobenius theorem requires for $v_{\lambda}$ :

$$
\left[v_{\lambda}, v_{\sigma}\right]=c_{\lambda \sigma \tau} v_{\tau} \text {. }
$$

Equation (C23) suggests the following form for the dual of the system $A$ :

$$
A^{\prime}=\left\{\begin{array}{l}
\bar{v}_{\lambda} \\
\phi_{\rho}=0,
\end{array}\right.
$$

$\bar{v}_{\lambda}$ being an extension of $v_{\lambda}$ to $T(X)$. It is obvious from the definition (C26) that $A^{\prime}$ has the same solutions as $A$.

Let us observe that $A^{\prime}$ is constructed by simply requiring the vectors $\bar{v}_{\lambda}$ to be weakly incident to the forms $\theta^{\alpha}$ and $d \phi_{\rho}$. Indeed we have

$$
\begin{gathered}
\left(v\left(j^{*} f\right)\right)(\rho)=v_{\rho}\left(j^{*} f\right)=\left(j_{*} v\right)_{j \rho}(f) \\
=\bar{v}_{j_{\rho}}(f)=\left(j^{*}(\bar{v}(f))\right)(\rho),
\end{gathered}
$$

that is

$$
j^{*}(\bar{v}(f))=v\left(j^{*} \theta\right),
$$

for any function $f$ on $X$. This can be extended to any 1-form:

$$
j^{*}\left(i_{\bar{v}} \theta\right)=i_{v}\left(j^{*} \theta\right) .
$$

Applying this result to $\bar{v}_{\lambda}$, we get

$$
j^{*}\left(\bar{v}_{\lambda} \theta^{\alpha}\right)=i_{v_{\lambda}}\left(j^{*} \theta^{\alpha}\right),
$$

and furthermore we have

$$
j^{*}\left(\bar{v}_{\lambda} \phi_{\rho}\right)=v_{\lambda}\left(j^{*} \phi_{\rho}\right)
$$

due to the definition of $V$.

Using the result of Appendix B, we may write $(\mathrm{C} 30)$ and (C31) as

$$
i_{\bar{v}_{\lambda}} \theta^{\alpha} \approx 0 \text {, }
$$

$$
i_{\bar{v}_{\lambda}} d \phi_{\rho} \approx 0 \text {. }
$$

In order to find the integrability condition for the system $A^{\prime}$, from $(\mathrm{C} 25)$ and using the property $(\mathrm{C} 28)$ we get

$$
\left[v_{\lambda}, v_{\sigma}\right]\left(j^{*} f\right)=c_{\lambda \sigma \tau} v_{\tau}\left(j^{*} f\right)
$$

or

$$
j^{*}\left(\left[\bar{v}_{\lambda}, \bar{v}_{\sigma}\right](f)\right)=j^{*}\left(c_{\lambda \sigma \tau}^{\prime} \bar{v}_{\tau}(f)\right),
$$

where

$$
j^{*} c_{\lambda \sigma \tau}^{\prime}=c_{\lambda \sigma \tau},
$$

that is,

$$
\left[\bar{v}_{\lambda}, \bar{v}_{\sigma}\right] \approx c_{\lambda \sigma \tau}^{\prime} \bar{v}_{\tau} .
$$

Equation (C33) is the integrability condition, that is, the Frobenius theorem, for the system $A^{\prime}$.

\section{APPENDIX D}

The transformation properties of the Hamilton-Jacobi function $S(x)$ under canonical transformations are known ${ }^{21}$; in the case of an infinitesimal transformation they have been given in particular by Bergmann. 22

Here we only recall the results.

Let us consider the situation described in Sec. III. If we know a complete integral of system $\phi_{\mu}(x, p)=0$ given by (3.37):

$$
\begin{aligned}
& S(x)=\phi\left(x^{\alpha}, c_{k}\right)+c \\
& (\alpha=0,1, \ldots, n, k=m+1, \ldots, n),
\end{aligned}
$$

we may ask what is the function

$$
S^{\prime}\left(x^{\prime}\right)=\phi\left(x^{\prime \alpha}, c_{k}\right)+c^{\prime}
$$

when we submit the system to the finite canonical transformation generated by the function $w(x, p)$, via the equations

$$
\begin{gathered}
p_{\alpha}^{\prime}=e^{w(x, p)_{*}} p_{\alpha}, \\
x^{\prime \alpha}=e^{u(x, p)_{*}} x^{\alpha},
\end{gathered}
$$

where the * operation is defined in Eq. (3.32).

More generally, we may consider the one-parameter canonical transformation

$$
\begin{aligned}
p_{\alpha}^{\prime}(\tau) & =e^{\tau \omega(x, p)} * p_{\alpha}, \\
x^{\prime \alpha}(\tau) & =e^{\tau u(x, p)_{*}} x^{\alpha} .
\end{aligned}
$$

The answer is given by the solution of the HamiltonJacobi equation:

$$
\frac{\partial S^{\prime}\left(x^{\prime}, \tau\right)}{\partial \tau}+w\left(x^{\prime}, \frac{\partial S^{\prime}\left(x^{\prime}, \tau\right)}{\partial x^{\prime}}\right)=0,
$$

with the boundary condition

$$
S^{\prime}\left(x^{\prime}, 0\right)=S(x) \text {. }
$$

In the case of an infinitesimal canonical transformation, by neglecting higher orders of $\tau$, we get ${ }^{21}$

$$
\delta S(x)=\tau w\left(x, \frac{\partial S}{\partial x}\right) .
$$

Equation (D7) shows in particular that, when the canonical transformation is generated by the constraints $\phi_{\mu}$, that is, for $w$ a given linear combination of $\phi_{\mu}$, the function $S$ is invariant.

As stressed by Bergmann, ${ }^{22}$ this has the consequence that $S$ is form-invariant under the invariance group (group generated by the constraints). In particular, the HamiltonJacobi theory does not require the setting of gauge conditions, which is, by the way, one of the main reasons for the relevance ascribed by the authors to a Hamilton-Jacobi approach to constrained systems in particle dynamics.

'Ph. Droz-Vincent, Lett. Nuovo Cimento 1, 839 (1969); 7, 206 (1973); Phys. Scripta 2, 129 (1970); Rep. Math. Phys. 8, 79 (1975); Ann. Inst. H. Poincaré 27, 407 (1977); Phys. Rev. D 19, 702 (1979); " $N$-Body Relativistic System," Ann. Inst. H. Poincaré 32, 377 (1980); L. Bel, "Meccanica Relativistica Predictiva," curso Depart. Fis. Teor., Barcelona Univ. UAB FT34, 1977; Ann. Inst. H. Poincaré 14, 189 (1971). L. Bel and J. Martin, Ann. Inst. H. Poincaré 22, 173 (1975); 33, 409 (1980). L. Bel and F. X. Fustero, Ann. Inst. H. Poincaré 25, 411 (1976). I. T. Todorov, "Dynamics of Relativistic Point Particles as a Problem with Constraints," Comm. JINR, EZ10125, Dubna, 1976; "Constraint Hamiltonian Dynamics of Directly Interacting Relativistic Point Particles," Lectures at the Summer School in Math. Phys. Bogazici Univ., Bebek, Istanbul, 1979, and at the 17th Winter School of Theoretical Physics, Karpacz, 1980; "Constraint Hamiltonian Approach to Relativistic Point Particles Dynamics," lectures at the ICTP, Trieste, 1980. V. V. Molotkov and I. T. Todorov, Comm. Math. Phys. 79, 11 (1981). S. N. Sokolov, Dokl. Akad. Nauk. SSSR 233, 575 (1977) [Sov. Phys. Dokl. 22, 198 (1977)]; Teor. Mat. Fiz. 36, 193 (1978) [Theor. Math. Phys. 36, 682 (1978)]. M. Kalb and P. Van Alstine, "Invariant Singular Action for the Relativistic Two-Body Problem: A Hamiltonian Formulation," Yale Report COO-3075-146, 1976. T. Takabayasi, Prog. Theor.

Phys. 57, 331 (1977); Suppl. Prog. Theor. Phys. 67, 1 (1979). K. Kamimura and T. Shimizu, Prog. Theor. Phys. 58, 383 (1977). K. Kamimura, Nuovo 
Cimento B 65, 181 (1981). D. Dominici, J. Gomis, and G. Longhi, Nuovo Cimento B 48, 152 (1978); Nuovo Cimento A 48, 257 (1978); A 56, 263 (1980). R. Giachetti and E. Sorace, Nuovo Cimento A 43, 281 (1978); Nuovo Cimento B 56, 263 (1980); Lett. Nuovo Cimento 26, 1 (1979). A. Komar, Phys. Rev. D 18, 1881, 1887, 3017 (1978); 19, 2908 (1979); "Relativistic Action-at-a-distance and quasiseparability," 24, 2330 (1981); F. Rohrlich, Ann. Phys. (N.Y.) 117, 292 (1979); 130, 350(1980); Phys. Rev. D 23, 1305 (1981). H. Sazdjian, Nucl. Phys. B 161, 469 (1979); Ann. Phys. (N.Y.) 136, 136 (1981). A. Kihlberg, R. Marnelius, and N. Mukunda, Phys. Rev. D 23, 2201 (1981); N. Mukunda and E. C. G. Sudarshan, Phys. Rev, D 23, 2210 (1981); E. C. G. Sudarshan, N. Mukunda, and J. N. Goldberg, Phys. Rev. D 23, 2218, 2231 (1981). L. Lusanna, "A Model for $N$ Classical Relativistic Particles," Nuovo Cimento A 64, 65 (1981); L. Lusanna, Nuovo Cimento B 65, 135 (1981). J. Gomis, J. A. Lobo, and J. M. Pons, Nuovo Cimento B 64, 316 (1981); Ann. Inst. H. Poincaré 35, 17 (1981). A. P. Balachandran et al., "Relativistic Particle Interactions: a Third World View," preprint, University di Napoli, "Unified Geometrical Approach to Relativistic Particle Dynamics," preprint, University of Texas at Austin, 1981; "Separability in Relativistic Hamiltonian Particle Dynamics," Phys. Rev. D 26, 3492 (1982); V. M. Penafiel, S. Orenstein, and K. Rafanelli, "Canonical Formalism for $N$ Interacting Relativistic Particles," preprint, Queens College, 1981. J. Samuel, "Relativistic Particle Models with Separable Interactions," Phys. Rev. D 26, 3482 (1982); "Constraints in Relativistic Hamiltonian Mechanics," 26, 3475 (1982).

${ }^{2}$ See also the Proceedings of the Workshop "Relativistic Action at a Distance: Classical and Quantum Aspects," edited by J. Llosa, Lecture Notes in Physics, Vol. 162 (Springer, Berlin, 1981).

${ }^{3}$ P. A. M. Dirac, Can. J. Math. 2, 129 (1950); 3, 1 (1951); Proc. R. Soc. London A 246, 326 (1958); Lectures on Quantum Mechanics, Belfer Graduate School, Yeshiva University, 1964.

${ }^{4}$ S. Shanmugadhasan, J. Math. Phys. 14, 677 (1973); E. C. G. Sudarshan and N. Mukunda, Classical Dynamics: A Modern Perspective (Wiley, New York, 1974); A. G. Hanson, T. Regge, and C. Teitelboim, Contributi del Centro Linceo Interdiscipl. di Scienze Mat. Fis, e Loro Applic., No. 22 (Lincei, Rome, 1976).

${ }^{5}$ For application of the Hamilton-Jacobi theory to constrained systems see A. Komar, Phys. Rev. D 18 (1978). For other applications, especially field theory, see, for instance: P. G. Bergmann, Phys. Rev, 144, 1078 (1966); H. A. Kastrup, Phys. Lett. B 70, 195 (1977); K. Kuchar, "Canonical Methods of Quantization," Utah Preprint UT 84 112, University of Utah, 1980, and references quoted therein.

${ }^{6}$ I. T. Todorov in Ref. 1.

${ }^{7}$ A. Komar in Ref. 1.

${ }^{8} \mathrm{G}$. Longhi in Ref. 2 .

${ }^{9}$ In order to guarantee the stability condition $\phi_{\rho}=0$, when $\phi_{\rho}$ belong to the first-class set, it is usually assumed that

$\left\{H_{c}, \phi_{\rho}\right\}=\sum_{\sigma} c_{\rho \sigma} \phi_{\sigma} \approx 0$.
${ }^{10}$ This assumption includes the case where $\left\|\partial \phi_{\rho} / \partial p_{\alpha}\right\|$ has rank $m+1$ as a particular but more physical case. Indeed, if this last condition does not hold, there will necessarily exist a relation between the coordinates $x^{\alpha}$, which usually contradicts the physical intuition, apart from particular systems like the rigid rotator. ${ }^{11}$ In our subsequent development of the theory, this will require an interchange of some momentum with the corresponding conjugate coordinate, via a canonical transformation. Thus we will maintain the hypothesis (2.9) for the sake of generality.

"See Hanson et al. in Ref. 4.

${ }^{12}$ Mathematical textbooks in which the theory of systems of partial differential equations (PDE) of the first order can be found, are, for instance: $\mathrm{E}$. Goursat, A Course in Mathematical Analysis (Dover, New York, 1959), Vol. II, Part Two, F. G. Tricomi, Equazioni a Derivate Parziali (Cremonese, Rome, 1957); L. E. P. Eisenhart, Continuous Groups of Transformations (Dover, New York, 1961); G. F. D. Duff, Partial Differential Equations (Univ. of Toronto Press, Toronto, 1956); J. Dieudonné, Treatise on Analysis (Academic, New York, 1974), Vol. IV, Chap. XVIII; Y. Choquet-Bruhat, C. DeWitt-Morette, and M. Dillard-Bleick, Analysis, Manifolds and Physics (North-Holland, Amsterdam, 1977), Chap. IV, Sec. C; R. Hermann, Interdisciplinary Mathematics (Math. Sci. Press, Brookline, MA, 1977), esp. Yol. XIV, Chaps. XV and XVI.

${ }^{13} \mathrm{Y}$. Choquet-Bruhat et al. in Ref. 12.

${ }^{14}$ Since Eqs. (2.14) determine $v$ only on the constraint surface, for the sake of simplicity we have chosen as a particular solution that given by Eqs. (2.17) and $(2.18)$ as true equalities in the whole space $R^{2 n+3}$. It is possible to verify that this particular choice does not entail any loss of generality.

${ }^{15}$ Goursat in Ref. 12.

${ }^{16} \mathrm{~J}$. A. Schouten and W. V. der Kulk, Pfaff's Problem and Its Generalizations /Clarendon, Oxford, 1949).

${ }^{17} \mathrm{See}$, for instance, Goursat and Tricomi in Ref. 12.

${ }^{18} \mathrm{This}$ point requires a further word. We have seen that $S$ as a function of $x$ does not exist. However, as mentioned at the beginning of this section, it can exist in some reduced space. Let us clarify this point. If we perform a canonical transformation on the original phase space, such that $2 s$ of the new canonical variables are algebraically equivalent to the second-class constraints, ${ }^{4}$ the remaining canonical variables define the so-called reduced phase space. Clearly, in this space, the set of equations for the Hamilton-Jacobi function reduces to $\phi_{\mu}^{*}=0$, with the above-mentioned set of $2 s$ variables put to zero. Since $\phi_{\mu}^{*}$ are first class, a Hamilton-Jacobi function will exist in the reduced configuration space.

${ }^{19}$ Sudarshan and Mukunda in Ref. 4.

${ }^{20} \mathrm{Pham}$ Man Quan, Introduction à la géométrie des variétés différentiables (Dunod, Paris, 1969).

${ }^{2}$ 'F. J. Testa, J. Math. Phys. 11, 2698 (1970); C. Lanczos, Variational Principles of Mechanics (Univ. of Toronto Press, Toronto, 1962).

${ }^{22} \mathrm{P}$. G. Bergmann in Ref. 5. 